IZA DP No. 5181

Does Bargaining Matter in the Small Firm Matching Model?

Olivier L'Haridon

Franck Malherbet

Sébastien Pérez-Duarte

September 2010 


\title{
Does Bargaining Matter in the Small Firm Matching Model?
}

\author{
Olivier L'Haridon \\ Greg-HEC and University Paris Sorbonne \\ Franck Malherbet \\ CARE - University of Rouen, \\ CECO - Ecole Polytechnique, $f R D B$ and IZA \\ Sébastien Pérez-Duarte \\ European Central Bank
}

\author{
Discussion Paper No. 5181 \\ September 2010
}

\author{
IZA \\ P.O. Box 7240 \\ 53072 Bonn \\ Germany \\ Phone: +49-228-3894-0 \\ Fax: +49-228-3894-180 \\ E-mail: iza@iza.org
}

\begin{abstract}
Any opinions expressed here are those of the author(s) and not those of IZA. Research published in this series may include views on policy, but the institute itself takes no institutional policy positions.

The Institute for the Study of Labor (IZA) in Bonn is a local and virtual international research center and a place of communication between science, politics and business. IZA is an independent nonprofit organization supported by Deutsche Post Foundation. The center is associated with the University of Bonn and offers a stimulating research environment through its international network, workshops and conferences, data service, project support, research visits and doctoral program. IZA engages in (i) original and internationally competitive research in all fields of labor economics, (ii) development of policy concepts, and (iii) dissemination of research results and concepts to the interested public.
\end{abstract}

IZA Discussion Papers often represent preliminary work and are circulated to encourage discussion. Citation of such a paper should account for its provisional character. A revised version may be available directly from the author. 
IZA Discussion Paper No. 5181

September 2010

\section{ABSTRACT}

\section{Does Bargaining Matter in the Small Firm Matching Model?*}

In this article, we use a stylized model of the labor market to investigate the effects of three alternative and well-known bargaining solutions. We apply the Nash, the Egalitarian and the Kalai-Smorodinsky bargaining solutions in the small firm's matching model of unemployment. To the best of our knowledge, this is the first attempt that has been made to implement and systematically compare these solutions in search-matching economies. Our results are twofold. First from the theoretical/methodological viewpoint, we extend a somewhat flexible search-matching economy to alternative bargaining solutions. In particular, we prove that the Egalitarian and the Kalai-Smorodinsky solutions are easily implementable and mathematically tractable within search-matching economies. Second, our results show that even though the traditional results of bargaining theory apply in this context, they are generally qualitatively different and quantitatively weaker than expected. This is of particular relevance in comparison with the results established in the earlier literature.

JEL Classification: $\quad$ C71, C78, J20, J60

Keywords: $\quad$ search and matching models, bargaining theory, Nash, Egalitarian, Kalai-Smorodinsky

Corresponding author:

Franck Malherbet

CARE - Université de Rouen

3, avenue Pasteur

76186 Rouen Cedex 1

France

E-mail: f.malherbet@gmail.com

\footnotetext{
* We are grateful to Pierre Cahuc, Olivier Charlot, Ashley Piggins, Fabien Postel-Vinay and Thorsten Upmann for their helpful comments and suggestions on an earlier version of this paper. The views expressed in this paper are those of the authors and should not be attributed to the European Central Bank, its Executive Board, or its management.
} 


\section{Introduction}

Search-matching models of unemployment have become the standard for studying aggregate labor markets. The existence of transaction costs on the labor market results in a local monopoly rent which is shared through bilateral bargaining between the firm and the worker. In the bulk of the search and matching literature, the conventional approach to determine wages is based on the use of a generalized Nash criterion (see for instance the surveys by either Mortensen and Pissarides, 1999 or Rogerson, Shimer and Wright, 2005). Although this criterion is used almost exclusively in economic applications, there is, a priori, no particular rationale rationale - either empirical or theoretical - to restrict to the Nash criterion alone.Consequently, one may wonder why the Nash solution is so popular. Usually, two lines of arguments are put to the fore. First, the Nash solution is flexible and mathematically tractable. Second, it has a natural strategic counterpart (see eg. Osborne and Rubinstein, 1990). These two lines of aargument, however, are not entirely satisfactory. As a matter of fact, the Nash solution has been challenged both from a theoretical and an empirical/experimental point of view. As emphasized by Thomson (1994), others solutions such as the Kalai-Smorodinsky (Kalai and Smorodinsky, 1975) and the Egalitarian (Kalai, 1977) solutions play a central role in bargaining theory and have, for the most part, been put aside. Furthermore, there are also strategic foundations for the other solutions (see Moulin, 1984 or Miyagama, 2002, among others). The prevalence of the Nash solution is all the more questionable in that a number of controlled lab-experiments have cast doubts on its relevance (see eg. Nydegger and Owen, 1974 or Nydegger, 1977). Additional experimental results go one step beyond and strongly support the Kalai-Smorodinsky (hereafter KS) solution over the Nash solution (Heckathorn, 1978). Despite this, discussions have remained mostly confined to the social choice/bargaining literature and, until recently, have been largely ignored in labor economics. Early and notable exceptions are McDonald and Solow (1981) and Alexander (1992) who offer characterizations of the Nash and the KS solutions in an efficient contract and a right-to-manage model, respectively. Recent contributions in labor economics suggest however that the Nash solution may be too simplistic. In this perspective, the recent contributions from Laroque and Salanié (2004) and Gerber and Upmann (2006) are of particular interest.

Laroque and Salanié (2004) use the KS solution to characterize the spread of the minimum wage to the wage distribution in an efficient contract model $\grave{a}$ la McDonald and Solow (1981). They show that under the Nash solution, a marginal increase in the the minimum wage does not affect the wage distribution due to a specific assumption ${ }^{1}$ which makes the bargained wages

\footnotetext{
${ }^{1}$ The independence to irrelevant alternatives axiom which has been the object of considerable controversy. We present the different solutions and axioms in a more detailed form in the core of this paper.
} 
independent of the minimum wage. The authors convincingly argue that such a property is counterfactual and that the KS solution is the one that is best suited from this particular standpoint. In what follows, we depart from these authors and consider only bargained wages, thereby excluding all others institutional wage agreements.

Gerber and Upmann (2006), again in an efficient contract model, challenge the innocuousness of the Nash solution. They argue that the labor market equilibrium hinges essentially on the bargaining solutions considered. In particular, they show that with the KS solution an increase in the reservation wage has an ambiguous effect on unemployment with the KS solution and that under reasonable parameter values employment may increase. This key result contradicts the traditional effect found with the Nash solution. They further demonstrate that alternative solution concepts such as the Egalitarian solution and the equal-loss solution (Chun, 1988) exhibit the same type of ambiguity. They conclude that this puzzling employment effect is by no mean a pathological/degenerate case and is more likely to be the rule than the exception.

Hence, this bring us to a more general question as regards the innocuousness of the bargaining solutions/assumptions in labor economics. Although the usual specification is Nash ex-post bargaining in the prevailing search-matching framework, the universality of this assumption needs to be questioned. Indeed, as emphasized by Mortensen and Pissarides (1999), wage determination is a major issue in the context of search equilibrium modeling. Existing literature (see Mortensen and Pissarides, 1999) has focused on alternative wage setting mechanisms (eg. insider-outsider divide, monopoly union or efficiency wage) but has neglected alternative bargaining solutions. To the best of our knowledge, this paper is the first paper that attempts to systematically characterize the way in which bargaining solutions affect equilibrium unemployment in a search and matching model of the labor market ${ }^{2}$. Taking the Nash solution as a benchmark and following Thomson (1994), we consider two alternative and prominent solutions, namely the Egalitarian and the KS solutions that are central to bargaining theory, within a somehow standard model $\grave{a}$ la Pissarides (2000). At this point, it is important to emphasize that our approach has been kept simple on purpose, in order to obtain, whenever possible, analytically tractable results and to focus on comprehensible mechanisms. This assumed parsimony is not deemed restrictive and enables us nonetheless to characterize a number of positive and normative properties of the three solutions.

Our central results are twofold. First from a theoretical/methodological viewpoint, we extend a somewhat flexible search-matching economy to alternative bargaining solutions. In particular and contrary to common beliefs, the Egalitarian and the KS solutions are proved to be easily

\footnotetext{
${ }^{2}$ Similar questions have been separately raised in search models of monetary exchange (see Aruoba, Rocheteau and Waller, 2007).
} 
implementable and mathematically tractable within search-matching economies. Second, our results show that even though the traditional results of bargaining theory apply in this context, they differ remarkably in both qualitative and quantitative terms as compared to the results established in the earlier literature (see for instance, Gerber and Upmann, 2006). More precisely, through the paper we contrast two economies: an economy with perfectly transferable utility which serves as our benchmark and an economy with a partially transferable utility only ${ }^{3}$. In the former case, we show that the three bargaining solutions boil down to an unique solution and yield a Pareto optimal resource allocation when the economy is constrained efficient. In the latter case, when utility is not perfectly transferable because of workers' risk aversion, the three solutions are proved to diverge and a first-best resource allocation is not feasible when wages are bargained over. Interestingly, the nonlinear discrepancy between the Nash and the KS solutions is proved to be small and this from both a qualitative and a quantitative viewpoint. This is not only the case in the neighborhood of linearity as might be expected, but also generalizes at high (and reasonable) levels of risk aversion for plausible parameter values. Of secondary interest, but nevertheless worth mentioning, is that under CRRA the Nash and the KS solutions yield similar outcomes when the Arrow-Pratt coefficient of risk aversion is equal to 0 and 2 , ie. for boundary values in the range accepted in the literature ${ }^{4}$. In general, the Nash and the KS solutions behave similarly under fairly mild conditions. Therefore, except for purposes based on the very axiomatic differences between bargaining solutions, the KS solution offers no significant improvement over the Nash solution for labor market analysis. Results are more contrasted as regards the Egalitarian solution. The difference between the Nash and the Egalitarian solutions is much more pronounced. This contrast owes much to the axiomatic difference between the two solutions, in particular as regards the scale invariance property. Finally, the three solutions are proved to behave identically following a change in the key structural parameters. This is analytically true for the solutions considered in the neighborhood of linearity and globally for the Nash and the Egalitarian solutions. However, because of the proximity between the Nash and the KS solutions, the results generalizes for the KS solution for a wide range of plausible parameter values. Hence our results again run counter to those previously established in the literature.

The outline of this article is as follows. Section 1 presents a stylized search model. Section 2 details the bargaining solutions under consideration. A particular emphasis is put on some

\footnotetext{
${ }^{3}$ Following Burdett and Wright (1998), transferable utility refers to the case where the frontier in utility is linear while non-transferable utility refers to the case where outside institutions or constraints yield discontinuity in the frontier. In this article, the frontier is nonlinear because of workers' preferences and because we do not introduce any additional constraints for discontinuity. To avoid any confusion between the continuous and discontinuous case, we say that utility is partially transferable when workers are risk-averse.

${ }^{4}$ See Chetty (2006).
} 
key axioms. Section 3 focuses on the key properties of the model when utility is perfectly transferable. Section 4 extends the model to the case of partially transferable utility. The properties established in section 3 are then discussed and illustrated in the case of partially transferable utility. Finally, section 5 concludes.

\section{The Model}

The benchmark model builds on a continuous-time search and matching framework. The core of the model is in many ways similar to the canonical textbook model of Pissarides (2000, Chapter 1), the objective being to remain as close as possible to a well-established framework except that alternative bargaining solutions are considered. Note however, that we will consider a slightly modified framework in the next-to-last section of this paper. For the remainder of the paper, however, we will restrict the analysis to the steady state.

\section{$1.1 \quad$ Main assumptions}

We consider an economy populated with a continuum of infinitely-lived and homogenous workers and a continuum of identical firms holding at most one vacancy. The size of the population is normalized to unity. All agents discount future payoffs at the rate $r>0$.

The labor market has matching frictions. Searching for a job and matching with a worker are costly activities. Frictions are captured by a customary matching function, $H$, with constant returns to scale. The flow of hires satisfies $H(\nu, u)$ where $\nu$ and $u$ indicate the number of vacancies and unemployed, respectively. Let $\theta \equiv \nu / u$ be the labor market tightness. The transition rate for vacancies is $q(\theta)$ with $q(\theta) \equiv H(\nu, u) / \nu=H(1,1 / \theta)$. Similarly, the flow out of unemployment is $\theta q(\theta)$ with $\theta q(\theta) \equiv H(\nu, u) / u=H(\theta, 1)$. The properties of the matching function imply that $q(\theta)$ and $\theta q(\theta)$ are decreasing and increasing in labor market tightness, respectively. Filled jobs are destroyed at a Poisson rate $s$. The evolution of the unemployment rate satisfies:

$$
\dot{u}=s(1-u)-\theta q(\theta) u
$$

In a steady-state, the unemployment rate is obtained by equating the flows out of unemployment, $\theta q(\theta) u$, to the flows in unemployment, $s(1-u)$, and verifies:

$$
u=\frac{s}{s+\theta q(\theta)}
$$

It is worth remarking here that the steady-state unemployment rate, $u$, is decreasing in the labor market tightness, $\theta$, ie. $\left.\frac{\partial u}{\partial \theta}\right|_{u}<0$. 


\subsection{Value functions}

Let $U$ and $E($.$) denote the lifetime expected utility of an unemployed worker and an employed$ worker, respectively. The associated value functions verify:

$$
\begin{aligned}
& r U=v(z)+\theta q(\theta)(E(w)-U), \\
& r E(w)=v(w)+s(U-E(w)) .
\end{aligned}
$$

Workers' utility stems from an instantaneous von Neumann and Morgenstern utility function, $v($.$) with the usual properties. According to eq. (3), an unemployed worker earns an instan-$ taneous real return $z$ and changes state on the labor market at rate $\theta q(\theta)$. In turn, eq. (4) indicates that a worker earns an income flow $w$ and changes state at rate $s$.

Let $V$ and $J($.$) denote the lifetime expected value of an open vacancy and an occupied job,$ respectively. The associated value functions verify:

$$
\begin{gathered}
r V=-\gamma+q(\theta)(J(w)-V), \\
r J(w)=y-w+s(V-J(w)) .
\end{gathered}
$$

Recruiting is costly. According to equation (5), advertising a vacant job incurs a flow cost $\gamma$. A vacancy changes state at a rate $q(\theta)$. Similarly, equation (6) states that a filled job yields an instantaneous profit $y-w$ and changes state at a rate $q(\theta)$.

\section{Bargaining}

In this section, we describe the bargaining problem related to the settlement of a contract between a worker and a firm. We first detail the set of feasible outcomes and then apply three standard bargaining solutions to the problem - the Nash, Kalai-Smorodinsky and Egalitarian solutions. It is important here to mention that two complementary approaches to bargaining coexist in the literature. The first one, initiated by Nash (1950), is axiomatic. Based on a set of behavioral properties, or axioms, it defines the class of solutions consistent with these properties, and is thus mainly normative. The second approach is strategic. It describes the necessary strategic conditions to reach an agreement in a non-cooperative game. In the present paper, we aim to analyze the equilibrium properties of the three different solutions. For the sake of parsimony, we restrict ourselves to the axiomatic approach and focus on the normative properties of the different solutions rather than on the specific non-cooperative bargaining conditions required to reach an agreement. It is worth noting nevertheless that the different solutions also have strategic foundations (see, eg. Moulin, 1984 or Miyagawa, 2002). 
Upon matching, a job-worker pair generates a local monopoly rent. This rent originates from the matching frictions on the labor market and implies that the expected total return from productive activities, $J+E$, is strictly greater than the total return from search, $U+V$. This rent is then shared through a constant wage rate resulting from a decentralized firm-worker bargaining. Assuming symmetric equilibria, the aggregation of match-specific wage rules leads to an aggregate wage equation. ${ }^{5}$

\subsection{The feasible set}

The bargaining problem can be described in a two-dimensional space of the expected lifetime utilities of both agents. In order to characterize it, two elements are necessary: the disagreement point and the set of feasible allocations obtained when an agreement is reached. The disagreement point is equal to the values of continued search for the firm and the worker, namely $d=(V, U)$. It is worth observing from (4) that a wage set at $w=r U$ makes the worker indifferent between employment and unemployment: $E(r U)=U$. Similarly, according to (6), a wage set at $w=y-r V$ makes the value of a job to the firm identical to the value of vacant job, hence $J(y-r V)=V$. Consequently, the disagreement point can also be written as $d=(J(y-r V), E(r U))$ and the wage $w$ must satisfy the following constraint in an agreement point: $y-r V \geq w \geq r U$. Let $\Omega$ define the set of allocations obtained when an agreement is reached; $\Omega$ is the set of feasible allocations:

$$
\Omega=\left\{x \in \Re^{2} \mid \exists w \in \Re, r U \leq w \leq y-r V, x \preceq X(w) \text { and } X(w) \succeq d\right\}
$$

where $X(w)=(J(w), E(w))$ for $r U \leq w \leq y-r V$ is the Pareto frontier, and where $(a, b) \preceq(c, d)$ if and only if $a \leq c$ and $b \leq d .(\Omega, d)$ is a standard two-agent bargaining problem where $\Omega$ is convex and closed, and the individually rational utility allocations are bounded. The following two lemma demonstrate that $(\Omega, d)$ satisfies these two properties:

Lemma 1. $\Omega$ is convex and closed.

Proof. The Pareto efficient allocations are given by (4) and (6). The Pareto-frontier is defined as the set of all points $(J, E(J))$ with $E(J)=[v(y-(r+s) J+s V)+s U] /(r+s)$. Since $v^{\prime}>0$ and $v^{\prime \prime}<0$, the first and second derivatives of $E(J)$ are negative, the Pareto frontier is strictly decreasing and strictly concave. Convexity of $\Omega$ follows. By continuity of $E$ and $J, \Omega$ is closed.

Lemma 2. The set of individually rational utility allocations $\{\delta \in \Omega \mid \delta \succeq d\}$ is bounded and there exists $\delta \in \Omega$ with $\delta_{j}>d_{j}$ for $j=1,2$.

\footnotetext{
${ }^{5}$ For an in-depth explanation, see Pissarides (2000, Chapter 1). For the remainder of the paper, and in order to minimize the notations, we will consider only symmetric equilibria.
} 
Proof. The maximum value from a job to the firm is reached when worker is paid her reservation wage $w=r U$. The maximum value from a job to the worker is reached when $w=y-r V$ so that he/she captures the entire surplus from the match. If $\delta \in \Omega$ is individually rational $(\delta \succeq d)$ then $u \preceq(J(r U), E(y-r V))$ and $\{\delta \in \Omega \mid \delta \succeq d\}$ is bounded. By (4) and (6), E(w) is increasing in $w$ and $J(w)$ is decreasing in $w$. There exists $\widetilde{w}=r U+\epsilon, \epsilon \in(0, y-r V-r U)$ such that $(J(\widetilde{w}), E(\widetilde{w})) \succeq d$. Then there exists $\delta \in \Omega$ with $\delta_{j}>d_{j}$ for $j=1,2$.

We now proceed with the exposure of the three key solutions. We restrict ourself to symmetric bargaining $^{6}$ and choose as a benchmark a case where utility is perfectly transferable. This case constitutes of the standard search and matching framework (see eg. Pissarides, 2000, and Rogerson et al., 2005). Technically, it amounts to assuming that the utility function is increasing and linear in its argument. We will relax this hypothesis in the next-to-last section. Each solution yields an aggregate wage equation. In equilibrium, combined with a job creation condition, the aggregate wage equation determines the equilibrium values for the labor market tightness, $\theta^{*}$, and for the wage, $w^{*}$. Finally, given these values, one obtains thanks to eq. (2) the steady state unemployment rate.

\subsection{The Nash solution}

The Nash solution (1950) is the most common solution to determine wages in the bulk of the literature on labor markets with frictions. It follows from the maximization of the expected present value of the job to the worker and to the employer, net of the value of searching for an alternative partner. The Nash solution $\delta^{N}$ to the problem defined by the set of feasible allocations $\Omega$ and the disagreement point $d$ is defined as:

$$
\delta^{N}(\Omega, d)=\left\{x \in \Omega \mid \exists w^{*}, x=X\left(w^{*}\right) \text { and } w^{*}=\underset{w}{\arg \max }(J(w)-V)(E(w)-U)\right\}
$$

The wage $w_{N}^{*}$ solves:

$$
w_{N}^{*}=\underset{w}{\arg \max }(J(w)-V)(E(w)-U)
$$

This solution is the only one that satisfies the four following axioms:

- Pareto-Optimality (PAR)

- Symmetry (SYM)

- Invariance to equivalent utility representation (INV)

- Independence of irrelevant alternatives (IIA)

\footnotetext{
${ }^{6}$ The results readily extend to asymmetric bargaining.
} 
The first two axioms, (PAR) and (SYM), are common to all the bargaining solutions we will consider. They are detailed in great depth inter alia in Osborne and Rubinstein (1990) and Thomson (1994). Roughly speaking, they indicate, respectively, that no party can be made better off without harming the other (PAR) and that the solution is invariant to a permutation in the players (SYM). The third axiom (INV), scale invariance or invariance to equivalent utility representation, implies that the solution is invariant under positive affine transformations. This property is inherited from the von Neumann-Morgenstern utility specification. It prevents basing compromises on interpersonal comparisons of utility. The fourth and last axiom is by far the most controversial $^{7}$. Generally speaking, the contraction independence or independence of irrelevant alternatives (IIA) states that the outcome of a bargain (in our context, the bargained wage) should remain the same if the set of feasible agreements shrinks and still encompasses the initial solution.

The Nash solution is represented on Figure 1. The solution to the bargaining problem satisfies a tangency condition between the Nash curve and the Pareto frontier.

\section{Figure 1 about here}

Under Nash bargaining, the aggregate equilibrium wage is defined by (see Appendix A):

$$
w_{N}^{*}=r U+\frac{1}{2}(y-r V-r U)
$$

\subsection{The Egalitarian solution}

The origin of this solution is reputedly difficult to trace back. Arguments in favour of this solution are detailed with attention in Kalai (1977). The solution is characterized by equal gains for both parties. This concept is central to social choice economics and aims at endowing each agent with exactly the same welfare. The solution does not preserve all the axioms from the traditional Nash solution. As briefly mentioned above, the contraction independence has came under a lot of criticism. In the Egalitarian case this axiom is replaced by a strong monotonicity axiom (MON). It should be noted that contrary to the Nash solution, this solution is not scale invariant. (see eg. Thomson (1994) for details). It follows that the Egalitarian solution is the only solution that satisfies the following axioms:

- Pareto-Optimality (PAR)

- Symmetry (SYM)

\footnotetext{
${ }^{7}$ The next two solutions do not use the Independence of irrelevant alternatives (IIA) axiom. See below for a detailed presentation of these solutions.
} 
- Strong monotonicity (MON)

The Egalitarian solution performs best from the viewpoint of monotonicity. The strong monotonicity (MON) axiom states that all players should benefit from an expansion of the feasible set irrespective of whether the expansion is biased toward a particular player. This solution satisfies:

$$
\delta^{E}(\Omega, d)=\left\{x \in \Omega \mid \exists w^{*}, x=X\left(w^{*}\right) \text { and and }\left(J\left(w^{*}\right)-V\right)=\left(E\left(w^{*}\right)-U\right)\right\}
$$

The Egalitarian solution is represented on Figure 2 and is given by the intersection between the Pareto frontier and the $45^{\circ}$ line.

\section{Figure 2 about here}

The aggregate equilibrium wage is then defined by (see Appendix A):

$$
w_{E}^{*}=r U+\frac{1}{2}(y-r V-r U)
$$

\subsection{The Kalai-Smorodinsky solution}

The Kalai-Smorodinsky solution (1975) is characterized by equal proportional concessions for both agents from their respective utopia points. For each agent, this utopia point is defined by the maximum utility attainable subject to the constraint that no agent should receive less than her status-quo utility.

$$
\delta^{K S}(\Omega, d)=d+\Lambda\left(x^{*}-d\right)
$$

where $\Lambda=\max \left\{\lambda \in \Re \mid d+\lambda\left(x^{*}-d\right) \in \Omega\right\}$ and where $x^{*}$, the utopia point, denotes the pair of best individual outcomes in $\Omega$ for each agent. From an axiomatic perspective, this solution is in many aspects similar to the Nash solution except as regards the contraction independence axiom. This axiom is replaced here with an individual monotonicity (IM) axiom. This solution is the only one that satisfies the following four axioms:

- Pareto-Optimality (PAR)

- Symmetry (SYM)

- Invariance to equivalent utility representation (INV)

- Individual Monotonicity (IM)

The individual monotonicity (IM) axiom states that an expansion of the feasible set biased toward a particular player always benefit him/her. This monotonicity axiom is therefore weaker than that of the Egalitarian case. 
In the particular case of the KS solution, the first difficulty we are confronted with stems from the definition of the utopian ideal. This definition appears to be crucial since it affects the very nature of the disagreement points for each player. As it has already been emphasized, the disagreement points are determined by the fall-back or status-quo position each player is facing. In our framework, these positions are given by the expected utility of unemployment for a worker and the expected value of a vacancy for a firm. Yet these two values are themselves affected by the expectations of the agents about the labor market equilibrium. In what follows, in the case of a failure both parties return to their searching activities and expect to earn or to pay the equilibrium aggregate wage. Such an expectation is encompassed in the utopia point, which is therefore defined as pragmatic. However, a more radical definition of utopia could have been retained. In such a case, a breakdown in the bargaining leads both parties to expect to earn or to pay the utopian wage in the next match, i.e. the highest possible wage for the worker and the lowest possible wage for the firm. However this case, which is defined as utterly utopian, is difficult to justify. As a matter of fact, this definition leads firms to expect the market wage to equal the reservation wage and conversely, workers to expect the market wage to equal productivity. As both expectations are degenerate labor market equilibria, we rwill focus solely on the more appealing pragmatic definition.

We therefore rewrite (4) and (6) to take into account the agents' most opportunistic expectation. Henceforth, $\widehat{X}^{k}$ will refer, by convention, to agent's $k$ utopian expectations for $X \in\{E, J\}$ and $k \in\{e, f\}$ where $e$ stands for employee and $f$ for firm. Similarly, $\widehat{w}^{k}$ refers to the utopian wage for agent $k$. The firm's utopia solves:

$$
r \widehat{J}^{f}=y-\widehat{w}^{f}+s\left[V-\widehat{J}^{f}\right]
$$

where $\widehat{J}^{f}$ denotes the firm's most opportunistic expectation when it pays the utopian wage $\widehat{w}^{f}$. The firm's utopia implies that the net surplus of the worker is nil. The firm appropriates all the rent from the match so that the utopian wage (for the firm) is exactly equal to the reservation wage $r U$. Formally, the wage is therefore equal to $\widehat{w}^{f}=r U$. On the worker's side, utopia solves:

$$
r \widehat{E}^{e}=\widehat{w}^{e}+s\left[U-\widehat{E}^{e}\right]
$$

where $\widehat{E}^{e}$ denotes the worker's most opportunistic expectations when he/she gets the utopian wage $\widehat{w}^{e}$. The worker's utopia implies that the net surplus of the firm is nil. Hence, the worker seizes all the rent from the match so that the utopian wage (for the worker) is exactly equal to $y-r V$. Formally, the wage is therefore equal to $\widehat{w}^{e}=y-r V$. The Kalai-Smorodinsky solution satisfies:

$$
\frac{J(w)-V}{\widehat{J}^{f}-V}=\frac{E(w)-U}{\widehat{E}^{e}-U}
$$


The KS solution is represented on Figure 3. This solution is determined by the intercept between the line that connects the disagreement point $d=(V, U)$ and the utopian ideal $\left(\widehat{J}^{f}, \widehat{E}^{e}\right)$ and the Pareto frontier.

\section{Figure 3 about here}

The aggregate equilibrium wage is then defined by (see Appendix A):

$$
w_{K S}^{*}=r U+\frac{1}{2}(y-r V-r U)
$$

\section{Labour Market Equilibria and Properties}

\subsection{Job creation curve (Jc)}

Firms enter the labor market until all profit opportunities from new jobs are exhausted. In equilibrium, the rent from a vacant job is zero and the free-entry condition, $V=0$, is satisfied. Using (5) and (6) together with the free-entry condition yields the traditional job creation equation:

$$
\frac{\gamma}{q(\theta)}=\frac{y-w}{r+s}
$$

Labour demand decreases in the expected or average wage $w$, in the interest rate $r$, in the job destruction rate $s$ and in the cost of a vacancy $\gamma$. Conversely, it increases in the labor productivity $y$. The job creation curve is downward sloping in the $(\theta, w)$-space, hence the negative partial derivative $\left.\frac{\partial \theta}{\partial w}\right|_{J c}<0$.

\subsection{Wage curves (wc)}

In the previous section, we derived three aggregate wage equations. The Nash $w_{N}^{*}$, the Egali$\operatorname{tarian} w_{E}^{*}$ and the Kalai-Smorodinsky $w_{K S}^{*}$ wage curves are defined by the relations (10), (12) and (17), respectively. Under each solution, the wage increases in the labor productivity, $y$, in the real return, $z$ and in the advertising cost, $\gamma$. The three wage curves are upward sloping in the $(\theta, w)$-space, hence the positive partial derivative $\left.\frac{\partial \theta}{\partial w}\right|_{w c^{j}}>0$, for $j \in\{N, E, K S\}$.

\subsection{Equilibria}

Definition 1. A labor market equilibrium with perfectly transferable utility is made up of the labor market tightness, $\theta^{*}$, defined by (18), and a wage equation, $w_{j}^{*}$, for all $j \in\{N, E, K S\}$, defined by one of (10), (12) or (17). In other words, the labor market equilibrium is determined in the $(\theta, w)$-space by: 
- the intercept of the curves defined by (10) and (18) if the wage is bargained according to the Nash criterion.

- the intercept of the curves defined by (12) and (18) if the wage is bargained according to the Egalitarian criterion.

- the intercept of the curves defined by (17) and (18) if the wage is bargained according to the Kalai-Smorodinsky criterion.

The labor market equilibria are represented on Figure 4. It is worth remarking that when utility is perfectly transferable, the three wage equations have exactly the same shape (upward sloping and linear) in the $(\theta, w)$-space ${ }^{8}$.

\section{Figure 4 about here}

\subsection{Properties}

Proposition 1. Equivalence. In a steady-state equilibrium with perfectly transferable utility, the three bargaining solutions collapse to a single aggregate wage equation. This equation verifies:

$$
w=\frac{1}{2} z+\frac{1}{2}(y+\gamma \theta)
$$

Proof. Straightforward, using the free entry condition, $V=0$, and noting that the reservation wage is equal to $r U=z+\gamma \theta$.

It follows that there is a single labor market equilibrium $\left(\theta^{*}, w^{*}\right)$ compatible with the three solutions that yields an unique equilibrium unemployment rate. Proposition 1 thus establishes a strict equivalence result between the three wage rules in the canonical search and matching model.

When the labor market has matching frictions, the tightness is not necessarily efficient due to trade externalities between firms and workers. Otherwise stated, one extra hiring firm makes workers better off and leaves other firms worse off. Conversely, one additional searching worker makes firms better off and leaves other workers worse off. In our benchmark economy, the objective of the benevolent social planner consists in maximizing total production with respect to $\theta$ subject to the same matching constraint on labor market flows as the decentralized economy.

\footnotetext{
${ }^{8} \mathrm{~A}$ formal characterization of the labor market equilibria is given in Appendix C.
} 
For the sake of simplicity, we restrict ourselves to the case where $r \rightarrow 0$, thereby ignoring transitional dynamics. The objective of the planner simplifies to ${ }^{9}$ :

$$
\max _{\{\theta, u\}} \Gamma=(1-u) y-u \theta \gamma
$$

subject to eq. (2). The instantaneous social output is made up of the market return of productive job-worker pairs, $(1-u) y$, minus the aggregate search costs $\gamma \nu=\theta u \gamma$. Two remarks must be made when utility is perfectly transferable. First, the planner is not concerned by distributional issues. Wages and unemployment benefits are consequently excluded from the social welfare function. Second, $\Gamma$, is similar here to a Bergson-Samuelson social welfare function and since the planner has no aversion to inequity (by assumption), $\Gamma$, is reduced to an Utilitarian welfare function.

Therefore the critical point here is to assess whether or not the decentralized (bargaining) equilibria is socially efficient, $i e$. whether the social welfare is maximized or not. In the canonical search and matching model, it is well established that a necessary condition for a decentralized equilibrium with Nash bargaining to be efficient, is given by the so-called Hosios-DiamondPissarides (HDP hereafter) condition. Technically, this condition implies that the elasticity of the matching function with respect to unemployment must be equal to the bargaining power of the worker. It translates into $\eta=\frac{1}{2}$ in our symmetric setup where $\eta$ is the elasticity of the matching function. The economy is said to be constrained efficient provided the HDP condition holds. By virtue of Proposition 1, when utility is perfectly transferable, this result generalizes to the Egalitarian and the KS wage rules. An additional condition for efficiency is that the equilibrium not be affected by unemployment benefits, $z$. It follows that social output is maximized under the three bargaining solutions when the HDP condition is fulfilled and $z=0$. The following proposition summarizes this point.

Proposition 2. Efficiency. When utility is perfectly transferable, $z=0$, and the economy is constrained efficient, the Nash, the Egalitarian and the Kalai-Smorodinsky solutions all maximize social welfare. It follows that job creation and unemployment are socially efficient whatever the bargaining solution considered.

Proof. See Appendix D.

Proposition 2 demonstrates that when congestion/search externalities are internalized in a decentralized equilibrium, then the market creates an efficient number of jobs and yields a socially optimal unemployment rate. For instance, Hosios (1990) argues in his seminal paper that that

\footnotetext{
${ }^{9}$ Note that we previously normalized the utility of leisure/home production to zero.
} 
under perfectly transferable utility, none of the results should depend on any particular rationalization for the wage ${ }^{10}$. Our results generalize Hosios' results to the Kalai-Smorodinsky and Egalitarian bargaining solutions.

To complete the comparison between the three bargaining solutions, we now turn to some classical comparative statics exercises. The critical point here is to check whether or not the results provided by Gerber and Upmann (2006) hold in the standard search and matching framework. Recall that these authors argue that under fairly mild conditions economic policy implications (eg. a change in the reservation wage) hinge entirely on the bargaining solution considered. The next proposition summarizes the comparative statics results for the key exogenous (policy) parameters.

Proposition 3. Comparative statics. Whatever the bargaining solution considered:

- The aggregate wage increases in the match productivity $y$ and in the real return $z$, and decreases in the vacancy cost $\gamma$, the interest rate $r$, and in the destruction rate $s$.

- The labor market tightness increases in the match productivity y, and decreases in the real return $z$, in the vacancy cost $\gamma$, in the interest rate $r$, and in the destruction rate $s$.

Proof. See Appendix E.

By virtue of Proposition 1, it is easy to conclude that modeling wage setting through the Nash, the Egalitarian or the Kalai-Smorodinsky bargaining solution does not affect the sign or the value of the derivative of the wage or the tightness with respect to the main exogenous variables. The usual results apply (see Pissarides, 2000). Comparative statics results are summarized in Table 4 of Appendix E.

Hence, the preceding three propositions stress the the strict equivalence between the bargaining solutions in the standard search and matching model when utility is perfectly transferable. This latter assumption is fairly standard and from this standpoint, using a solution rather than another appears to be neutral. This obviously runs counter to the arguments developed by Gerber and Upmann (2006). Where does the divergence between the two models comes from? We will examine this issue in the next section.

\footnotetext{
${ }^{10}$ In his seminal article, Hosios (1990) considers the Nash solution only under perfectly transferable (linear) utility. See Hosios (1990) p. 283.
} 


\section{Matching Under Partially Transferable Utility}

So far, we have restricted the analysis to the case of perfectly transferable utility between the firms and the workers. In the previous development, such an assumption consists in assuming a linear utility function for each player in the bargaining games. This hypothesis is fairly standard in the search and matching literature (see eg. Pissarides, 2000 and Rogerson et al., 2005), and amounts to assuming complete financial markets where workers may fully insure against income variation and unemployment hazard. In this section, we relax this hypothesis and consider the case where risk averse workers have no access to financial markets. In this slightly modified framework, we characterize the three different solutions when utility is partially transferable ${ }^{11}$ and then we proceed to the comparison with the benchmark economy. It is worth noting that the job creation equation (18) is invariant to workers' preferences. For that reason and for the sake of parsimony, we impose from the start the free entry condition so that the value of a vacancy is nil, $V=0$.

\subsection{Equilibrium Wages}

Workers' preferences are now represented with a concave instantaneous von Neumann and Morgenstern utility function. Risk averse workers want to smooth utility across states. Within the scope of expected utility and when the firm and the worker bargains on wages, any increase in the degree of the worker's risk aversion strengthens the firm's position in the negotiation. Risk aversion reduces the set of feasible allocations $\Omega$ and modifies the shape of the Pareto frontier as depicted on the figures below. Note that it is also possible to alter the shape of the Pareto frontier by considering other institutional wage setting agreements. However, the assuming of risk aversion is more adapted to our model and is of particular relevance when dealing with eg. optimal unemployment insurance policy (see for example Fredriksson and Holmlund, 2001 or Coles and Master, 2006). In addition and as shown by Burdett and Wright (1998), it should be kept in mind that two-sided search with non-transferable or partially transferable utility may lead to multiple equilibria. However, the simple per period payoff or utility assumed in this paper provides a sufficient condition for uniqueness and rules out the possibility of multiple equilibria (see for instance Burdett and Wright, 1998, Proposition 2, for a formal statement).

Nash: The solution to the bargaining game still satisfies a tangency condition between the Nash curve and the Pareto frontier. Under Nash bargaining with non transferable utility, the

\footnotetext{
${ }^{11}$ The case of partially transferable utility has recently attracted much attention in search equilibrium models of the marriage market - see for instance Coles and Burdett (1997) and Burdett and Wright (1998)- but none of these papers have considered solution concepts other than that of Nash.
} 
aggregate equilibrium wage is implicitly defined by (see Appendix A for details):

$$
\frac{v(w)-v(z)}{v^{\prime}(w)}=y-w+\gamma \theta
$$

\section{Figure 5 about here}

The Nash solution with partially transferable utility is represented in Figure 5. The aggregate wage is set so as to equalize the workers' net utility gains (the left hand side) and the firms' profits (the right hand side). Workers' attitude toward risk has a direct impact on the left hand side of the wage equation and reduces the utility gap between wage and real return $z$. Thus, as the worker's risk aversion increases, the firm is able to hoard a greater share of the surplus and bargains over a smaller wage.

Egalitarian: Under the Egalitarian solution the aggregate equilibrium wage is then implicitly defined by (see Appendix A for details):

$$
v(w)-v(z)=y-w+\gamma \theta
$$

A straight comparison between (21) and (22) clearly shows the importance of the scale invariance property. Under the Nash solution, the workers' net utility, $v(w)-v(z)$, is normalized (scaled) by the wage's marginal utility, $v^{\prime}(w)$. This produces scale invariance to affine transformations of utility in the classical Arrow-Pratt sense. Under the egalitarian solution, the workers' net utility is not normalized. Accordingly, the solution depends on the marginal utility of the outcome, $i e$. on the utility's scaling. It is therefore necessary to assume a given arbitrary normalization for utility. In this case, we assume that the workers agree with the firm on the marginal utility value of labor productivity $y$, hence $v^{\prime}(y)=1$. The following lemma and propositions reassess the equivalence result when workers are risk averse.

Lemma 3. When utility is partially transferable (workers are risk averse), the Nash and the Egalitarian solutions are no longer equivalent.

The non equivalence between the two solutions originates from the scale invariance property of the Nash solution. Put differently, when workers are risk neutral, the marginal utility of the wage is equal to unity and the two solutions collapse to a single one. This does not hold true when workers are risk averse.

Kalai-Smorodinsky: Under the KS solution the aggregate equilibrium wage is implicitly defined as a function of the reservation wage $r U$ (see Appendix A for details):

$$
\frac{v(w)-r U}{v(y)-r U}\left(y-v^{-1}(r U)\right)=y-w
$$




\section{Figure 6 about here}

The Kalai-Smorodinsky with non transferable utility is represented in Figure 6. It is worth remarking that homogeneity in $v($.$) of equation (23) ensures that the Kalai-Smorodinsky solution$ remains scale invariant. In order to compare the Kalai-Smorodinsky and the Nash solutions, it is convenient to rewrite $(21)$ as $^{12}: \frac{v(w)-r U}{v^{\prime}(w)}=y-w$. Comparing the latter expression with (23), it is easy to note that the two solutions differ.

Lemma 4. When risk-averse workers' utility is partially transferable, the Nash and the KalaiSmorodinsky solutions are in general no longer equivalent.

More accurately, the non equivalence originates from the discrepancy between $v^{\prime}(w)$ and $\frac{v(y)-r U}{y-v^{-1}(r U)}$, and there is, a priori, no reason for these two expressions to be identical. Note however that if $y$ and $v^{-1}(r U)$ (which define the upper and lower bounds of feasible wages) are sufficiently close, then $\frac{v(y)-v\left(v^{-1}(r U)\right)}{y-v^{-1}(r U)}$ is a first order approximation of $v^{\prime}(x)$ at the reservation wage $x=v^{-1}(r U)$. If the wage under the Nash solution is sufficiently close to the reservation wage, the difference between the two solutions tends to vanish. In other words, the discrepancy between the two solutions is conditioned by their position within the range of feasible wages and the curvature of the utility function. Finally, putting all elements together and remarking that the egalitarian solution can be rewritten as $v(w)-r U=y-w$, it is possible to summarize the preceding results.

Proposition 4. Equivalence. When utility is partially transferable, the Nash, the Egalitarian and the Kalai-Smorodinsky solutions differ and the equivalence result collapses.

Beyond that we cannot say much more about the differences between the three bargaining solutions from a qualitative standpoint, at least in their general forms. The key therefore is to evaluate the quantitative relevance of these differences which are themselves determined by the shape of the Pareto frontier. We will discuss these considerations in the next section and return for the moment to the efficiency and comparatives statics issues.

\subsection{Labor Market Equilibria}

In the benchmark model, whatever the bargaining solution, the wage curves all have the same shape, ie. they are upward sloping and linear in the $(\theta, w)$-space. When utility is partially transferable, things turn out to be different. We have already established that the three solutions are no longer equivalent. We can also characterize the difference between the solutions. The

\footnotetext{
${ }^{12}$ Under the Nash solution, when utility is partially transferable, the reservation wage rewrites as $r U=v(z)+$ $\theta \gamma v^{\prime}(w)$. Using this latter expression together with (21), the expression in the core of the text follows. For further details see Appendix A.
} 
shapes of the wage curves are now different. More precisely, when utility is partially transferable, all of the wage curves are upward sloping and the Nash, the Egalitarian and the KS wage curves are respectively convex, concave and convex in the $(\theta, w)$-space ${ }^{13}$. The Job creation curve is unaffected in our updated framework. A set of possible labor market equilibrium when utility is partially transferable is represented in Figure 7.

\section{Insert Figure 7 about Here}

Note that with partially transferable utility, it is not possible to establish a systematic hierarchy in the wage or in the labor market tightness. As a consequence, the labor market equilibria may be ordered differently than in figure 7 although the current figure is consistent with with the main results presented in section 4.4 .

\subsection{Properties}

With partially transferable utility, the production criterion as emphasized in the linear case is non longer equivalent to the Utilitarian criterion. As in the benchmark model, we restrict to the case $r \rightarrow 0$. Following eg. Fredriksson and Holmlund (2001) or Lehmann and van der Linden (2007), we assume that a benevolent social planner will choose the optimal allocation that maximizes an Utilitarian criterion subject to matching constraints and to an aggregate resource constraint. The benevolent social planner controls the tightness, the wages and the unemployment benefits. The social planner's objective simplifies to:

$$
\max _{\{\theta, u, w, z\}} \Gamma=(1-u) v(w)+u v(z)
$$

subject to eq. $(2)$ and $(1-u) w+u z=(1-u) y-\theta u \gamma$. The welfare function, $\Gamma$, is made up of the instantaneous utility of employed and unemployed workers weighted by their respective mass. The first constraint is similar to the one used in the benchmark model and underlines the fact that the planner faces the same matching constraints as the decentralized economy. The last constraint simply states that a feasible allocation must satisfy an aggregate resource constraint.

The planner problem is detailed in Appendix D. A key property of the first-best allocation is perfect insurance. The planner sets wages and unemployment benefits in such a way that $v^{\prime}(w)=v^{\prime}(z)$. This perfect insurance property implies that workers are wage takers. Hence as it has already been shown by eg. Lehmann and van der Linden (2007), unless workers have no bargaining power (ie. the workers are paid their reservation wage), the first-best optimum

\footnotetext{
${ }^{13}$ Formal proofs are provided in Appendix C.
} 
cannot be reached through decentralized bargaining. Of course, none of the wage agreements in this paper satisfies such a rule. The next proposition summarizes the arguments when utility is partially transferable.

Proposition 5. Efficiency. When utility is partially transferable and the economy is constrained efficient, the Nash, the Egalitarian and the Kalai-Smorodinsky solutions do not maximize social welfare unless workers are assumed to be wage takers. It follows that in the general case, job creation and unemployment are socially inefficient whatever the bargaining solution considered.

Proof. see Appendix D.

Propositions 2 and 5 characterize the first-best allocation in the two specifications of the model, although the formal conditions differ significantly. As discussed by Lehmann and van der Linden (2007), the difference stems from the fact that the planner is not concerned with distributional issues when utility is perfectly transferable. As a consequence, there is a multiplicity of first-best allocations, characterized by a combination of $w$ and $z$, maximizing total production net of the vacancy costs. The constrained efficient benchmark economy with $z=0$ corresponds to a particular optimum among all the first-best allocations. Contrary to the transferable case, when utility is partially transferable, the planner pays attention to the manner in which output is distributed among workers and perfectly insures workers against income risk. This perfect insurance property makes bargaining considerations irrelevant from a first-best perspective. Consequently any bargained wage would lead to inefficient outcomes. In what follows, we focus on the second-rank characteristics of the bargaining solutions using the welfare function $\Gamma$.

Under Nash or Egalitarian bargaining, it is easy to establish direct comparative statics inferences. The next proposition shows that proposition 3 easily extends to the case of partially transferable utility for both bargaining solutions.

Proposition 6. Comparative statics: Nash and Egalitarian. Given Nash or Egalitarian bargaining:

- The equilibrium wage is increasing in the match productivity $y$ and in the real return $z$, and decreasing in the vacancy cost $\gamma$, the interest rate $r$, and in the destruction rate $s$.

- The labor market tightness is increasing in the match productivity y, and decreasing in the real return $z$, in the vacancy cost $\gamma$, in the interest rate $r$, and in the destruction rate $s$. 
Proof. See Appendix E.

Hence, it turns out that from a qualitative viewpoint the comparative statics effects are identical for the two solutions considered. Recalling that the unemployment rate is decreasing in the labor market tightness, Proposition 6 shows that equilibrium employment increases in $y$ and decreases in $z, \gamma, r$ and $s$. In particular, it is worth noting that a rise in the real return $z$ (which increases the reservation utility $r U$ and subsequently the reservation wage $\left.v^{-1}(r U)\right)$ unambiguously raise the unemployment rate in both cases. This result runs counter to the arguments proposed by Gerber and Upmann (2006) as regards the Egalitarian solution ${ }^{14}$.

The comparative statics properties under the KS solution are harder to characterize. As a matter of fact, the equilibrium wage is an implicit function of the reservation utility $r U$, which is, in itself, a function of the wage and the labor market tightness. Formally, the reservation wage satisfies:

$$
r U=\frac{r+s}{r+s+\theta q(\theta)} v(z)+\frac{\theta q(\theta)}{r+s+\theta q(\theta)} v(w)
$$

Together with the job creation condition (18) and the wage equation (23), equation (25) defines the labor market equilibrium under the KS solution. Unfortunately, in its general form the KS solution is not easily tractable. To derive analytical results, we proceed to a first-order Taylor expansion in the neighbourhood of the linear/transferable case. Consequently, it must be borne in mind that the results apply essentially to a reasonably low degree of worker risk aversion. However, it can be demonstrated that the Nash and the KS solutions are indiscernible in a firstorder approximation ${ }^{15}$. We show in Appendix E that the Nash and the KS solutions behave identically under fairly mild conditions. The following proposition summarizes the results:

Proposition 7. Comparative statics: Kalai-Smorodinsky. Given the Kalai-Smorodinsky solution in the neighbourhood of the transferable utility case and under fairly mild conditions:

- The equilibrium wage is increasing in the match productivity $y$ and in the real return $z$, and decreasing in the vacancy cost $\gamma$, the interest rate $r$, and in the destruction rate $s$.

- The labor market tightness is increasing in the match productivity $y$, and decreasing in the real return $z$, in the vacancy cost $\gamma$, in the interest rate $r$, and in the destruction rate $s$.

Proof. See Appendix E.

\footnotetext{
${ }^{14}$ For instance, see Gerber and Upmann (2006, pp. 171-172).

${ }^{15}$ See Appendix B for a formal proof. The two solutions differ however in the second-order. In consequence, the quantitative differences between the two solutions are likely to be second-order differences which is also consistent with the discussion in the previous section.
} 
Proposition 7 shows that from a qualitative standpoint the Nash and the KS solutions behave identically in the neighborhood of the linear/transferable case. Again, this contradicts Gerber and Upmann (2006). When utility is non transferable, the Nash and the KS solutions are no longer equivalent however an accumulation of evidence suggests that the discrepancy between the two solutions is small. In the next section, we proceed to a series of numerical exercises in order to further evaluate the generality of our arguments.

\subsection{Quantitative Implications}

It has been established so far that with partially transferable utility the equivalence result between the three bargaining solutions vanishes. In addition, it has been shown that in general the solutions behave identically at least in the neighborhood of linearity. The purpose here is to determined the qualitative and quantitative implications of the model in its general form when utility is partially transferable. We proceed in three steps. First, we parameterize the model and compare the three solutions. Second, we search for the degree of risk aversion that maximizes the distance between the solutions, in particular as regards the Nash and the KS solutions. Third, we evaluate the sensitivity of the model to variations in the key exogenous parameters.

Numerical Illustrations: The baseline parameter values are chosen with two criteria in mind: (i) the values of the parameters themselves have to be realistic and coherent with the values usually chosen in the literature; (ii) the values of the endogenous variable that follow from these parameters also have to be reasonable. The parameters used here are in the range of those usually chosen in the matching literature (see eg. Shimer, 2005). We normalize the time period to be a quarter. The discount rate, $r$, is set to $1.25 \%$. A matching function of the Cobb-Douglas form is assumed such that $M(u, v)=k u^{\vartheta} v^{1-\vartheta}$, where $k$ is a mismatch parameter, $\vartheta$ and $1-\vartheta$ are the elasticities of the matching function with respect to search inputs. We assume $\vartheta$ to be equal to $\frac{1}{2}$ (see eg. Petrongolo and Pissarides, 2001). As is traditional in the literature, parameter $k$ may be used as a calibration parameter to pin down the equilibrium unemployment rate. We set this parameter to unity. The values of leisure and the recruiting costs are set to $z=0.4$ and $\gamma=0.27$, respectively. The job productivity is normalized to unity. We assume a CRRA utility function of the form:

$$
v(x)= \begin{cases}\frac{x^{1-\sigma}}{1-\sigma} & \sigma \geq 0, \sigma \neq 1 \\ \ln x & \sigma=1\end{cases}
$$

where $\sigma$ is the coefficient of relative risk aversion in the Arrow-Pratt sense. We take the symmetric Nash bargaining solution under transferable utility as a benchmark. Note that in the benchmark model the economy is constrained efficient since the bargaining power of the work- 
ers is equal to the elasticity of the matching function. The baseline parameter values and the resulting endogenous variables are reported in Table 1.

\begin{tabular}{lcccccc}
\hline \hline Parameters & $y$ & $s$ & $z$ & $\gamma$ & $r$ & $k$ \\
& 1 & 0.1 & 0.4 & 0.27 & 0.0125 & 1 \\
\hline Endogenous variables & $\theta$ & & $w$ & $u$ & \\
& 1.91 & 0.9580 & $6.75 \%$ & \\
\hline \hline
\end{tabular}

Table 1: Baseline parameter values and equilibrium labor market values with symmetric Nash Bargaining and transferable utility.

Table 2 presents the labor market equilibrium values for the three bargaining solutions. We contrast two cases in terms of risk aversion: low risk aversion $(\sigma=0.5)$ and high risk aversion $(\sigma=1.5)$. In the first case the curvature of the utility function is small whereas in the second the curvature is more pronounced. For each solution and each risk level, we report the equilibrium values for the wage, $w$, the tightness, $\theta$, the unemployment rate, $u$, and a measure of the Welfare, $\Gamma$, which here is similar to a Utilitarian criterion ${ }^{16}$.

\begin{tabular}{lcccc}
\hline \hline \multicolumn{1}{c}{$\sigma=0.5$} & $w$ & $\theta$ & $u$ & $\Gamma$ \\
\hline Nash & 0.9538 & 2.3183 & $6.16 \%$ & 1.9156 \\
Egalitarian & 0.9532 & 2.3738 & $6.09 \%$ & 1.9156 \\
Kalai-Smorodinsky & 0.9538 & 2.3180 & $6.16 \%$ & 1.9156 \\
\hline \multicolumn{1}{c}{$\sigma=1.5$} & $w$ & $\theta$ & $u$ & $\Gamma$ \\
\hline Nash & 0.9429 & 3.5281 & $5.05 \%$ & -2.1093 \\
Egalitarian & 0.9404 & 3.8527 & $4.85 \%$ & -2.1095 \\
Kalai-Smorodinsky & 0.9430 & 3.5274 & $5.06 \%$ & -2.1093 \\
\hline \hline
\end{tabular}

Table 2: Equilibrium values for the three bargaining solutions and for two risk aversion's levels ( $\sigma=0.5$ is low risk aversion; $\sigma=1.5$ is high risk aversion)

The first noticeable result is that for any degree of risk aversion, the quantitative difference between the Nash and the KS solution is immaterial. With low risk aversion, the small curvature of the utility function makes the difference between the two solutions non-existent (for further details see the discussion in subsection 4.1). Therefore when utility is partially transferable and when a low degree of risk aversion is involved, the equivalence result remains. With high risk aversion, the large curvature of the utility function creates a second-order difference between the two solutions. However, for reasonable parameter values, the difference as regards the labor market is negligeable. Hence, although the two solutions differ (widely) from a theoretical viewpoint when utility is partially transferable, the quantitative discrepancies appear insignificant.

The results diverge more significantly as regards the Egalitarian solution. As detailed in subsection 4.1, the bargaining outcome from the Egalitarian solution depends on marginal utility

\footnotetext{
${ }^{16}$ Formally this criterion satisfies: $\Gamma=(1-u) r E+(1-u) r J+v r V+u r U$.
} 
and differs from the other two scale invariant solutions. For any degree of risk aversion, the wage and the tightness are lower and higher, respectively, under the Egalitarian solution, hence a lower unemployment rate. Perhaps surprisingly, the welfare tends to be smaller despite a lower unemployment rate. For the sake of clarity, let us recall that the instantaneous Utilitarian criterion may be written as as $(1-u)(y-w)+(1-u) v(w)+u v(z)-u \theta \gamma$. As can be inferred from Table 2, the Egalitarian solution is more responsive in the tightness than the Nash or the KS solutions. It follows that the last term matters more, hence a more pronounced effect on welfare. Finally and perhaps trivially, one can note that whatever the bargaining solution considered, a higher degree of risk aversion implies a lower wage and a higher labor market tightness.

Maximum Difference: We now proceed with the analysis of the model for admittedly plausible values of $\sigma$ in the range $[0 ; 2]$. Chetty (2006), for instance, finds bounds on the Arrow-Pratt coefficient of relative risk aversion $\sigma$ and estimates that this coefficient should be smaller than 2 and is supposedly close to 1 . Over the range considered, the difference between the Nash and the Egalitarian solutions is monotonic whereas the difference between the Nash and the KS solutions is non-monotonic ${ }^{17}$. Here we restrict to the latter case. It has been established that although these two solutions differ from a theoretical viewpoint they are, under partially tranferable utility, arguably close. We search for the maximum discrepancy between the two solutions for the model detailed above. One of the more noteworthy results here is that with a CRRA utility function the Nash and the KS solutions coincide at the upper and the lower bound of the interval considered. The following remark highlights this result.

Remark 1. With a CRRA utility function, the Nash and the Kalai-Smorodinsky solutions are reduced to a single solution when workers are risk neutral $(\sigma=0)$ or strongly risk averse $(\sigma=2)$.

Proof. For $\sigma=0$ direct by virtue of Proposition 1 . For $\sigma=2$, we get $v(x)=-1 / x, v^{\prime}(x)=1 / x^{2}$ and $v^{-1}(x)=-1 / x$. Replacing in the wage equations as defined in Appendix A for partially transferable utility, the solution to the bargaining problem verifies $y=-r U w^{2}$.

The wage gaps $w^{K S}-w^{N}$ (panel a) and $w^{E}-w^{N}$ (panel b) are plotted below for $\sigma \in[0 ; 2]$. Over the range considered and for plausible parameter values, we have $w^{K S} \geq w^{N}$ and $w^{N} \geq w^{E}$ which is consistent with the labor market equilibria depicted in figures 4 and 7 . On the first panel, the wage gap is bell-shaped and maximized for $\sigma=1.28$. Note that the max is quasiinvariant to small change in the key parameters for the model specification we have chosen. Two remarks can be made here. First, the quantitative parameterizations of the model unambiguously shows that the bargained wage under the Kalai-Smorodinsky solution is not always higher that

\footnotetext{
${ }^{17}$ See Appendix B for formal details. Numerical illustrations are given in Figure 8 below.
} 
the bargained wage under the Nash solution. This specific ranking is restricted to a precise range of relative risk aversion coefficients, namely $[0 ; 2]$. Outside this range, the ranking may be reversed. Second, the deviations from the perfectly transferable case do not yield any systematic or monotonic relationship between risk aversion and the wage gap.

\section{Insert Figure 8 about here}

Steady-State Elasticities: The purpose here is to underline the quantitative effects of a change in some key exogenous parameters. In particular, we are interested in the comparative-statics effects of the Kalai-Smorodinsky solution from both a qualitative and quantitative point of view. We evaluate the responsiveness of the wage and the labor market tightness to a variation in: (i) the labor productivity, $y$; (ii) the unemployed real return, $z$; (iii) the cost of a vacancy; (iv) the interest rate, $r$; and $(v)$ the job separation rate, $s$. More precisely, we calculate the percentage change in the endogenous variable in response to a $10 \%$ increase in the exogenous parameter. It follows that $\varepsilon^{y / x} \equiv \frac{d \log y}{d \log x}, \quad \forall x \in\{y, z, \gamma, s, r\} \quad$ and $\forall y \in\{\theta, w\}$. Table 3 summarizes the steady-state elasticities for the wage and the labor market tightness following a change in a key parameter when the difference between the Nash and the KS solutions is maximum for $\sigma=1.28$ as evaluated above. Note that with these two criteria we stress numerical configurations that should exacerbate the discrepancies between the bargaining solutions especially as regards the Nash and the KS solutions.

\begin{tabular}{lccccc}
\hline \hline \multicolumn{1}{c}{$\varepsilon^{w / y}$} & $y$ & $z$ & $\gamma$ & $r$ & $s$ \\
\hline Nash & 0.9866 & 0.0441 & -0.0291 & -0.0067 & -0.0529 \\
Egalitarian & 1.0346 & 0.0484 & -0.0321 & -0.0073 & -0.0584 \\
Kalai-Smorodinsky & 0.9866 & 0.0441 & -0.0291 & -0.0066 & -0.0529 \\
\hline \multicolumn{1}{c}{$\varepsilon^{\theta / y}$} & $y$ & $z$ & $\gamma$ & $r$ & $s$ \\
\hline Nash & 2.4154 & -1.4380 & -1.1049 & -0.0239 & -0.1908 \\
Egalitarian & 0.9365 & -1.5040 & -1.0614 & -0.0140 & -0.1119 \\
Kalai-Smorodinsky & 2.4153 & -1.4376 & -1.1051 & -0.0239 & -0.1912 \\
\hline \hline
\end{tabular}

Table 3: Steady-State elasticities for a $10 \%$ increase in the key exogenous parameters.

From Table 3, it can be remarked that the comparative statics results of the the KS solution in the neighborhood of linearity as presented in Proposition 7 generalize under reasonable parameters values in the neighborhood of linearity. It follows that the three bargaining solutions yield the very same qualitative results. Furthermore, even in a configuration where the discrepancies between the Nash and the KS solutions should be exacerbated, the difference between the two solutions appears to be intangible. This last point corroborates our previous results. Finally, the Egalitarian solution appears to be more responsive to the change of the key parameters. In particular, the elasticities of the wage and the tightness to the labor productivity and the job 
separation rate are magnified in comparison with the Nash and the KS solutions. Hence from a macroeconomic viewpoint only the Egalitarian solution is likely to change the labor market responses to aggregate turbulence.

\section{Conclusion}

In this article we use a stylized model of the labor market to investigate the effects of three alternative and prominent bargaining solutions. We studied the Nash, the Egalitarian and the Kalai-Smorodinsky bargaining solutions in a small firm's matching model of unemployment. Our results are twofold. First, from a theoretical point of view, this paper is to the best of our knowledge the first contribution that attempts to implement and systematically compare the outcome of the different solutions in a search and matching model of the labor market. Second, we establish a series of results arising out of the different bargaining solutions considered. Our results show that even though the traditional results of bargaining theory apply in the context of search-matching economies, they are quantitatively weaker than expected. In particular, we found no difference in labor market outcomes between the Nash and the KS solutions. This suggests that the results advocated by eg. Gerber and Upmann (2006) in a McDonaldSolow efficient contract model do not generalize to dynamic search-matching economies. A main consequence is that the policy implications are not very sensitive to the choice of the bargaining solution used to represents wage bargains on the labor market. A secondary consequence is that the tractability of the Nash solution comes at low cost in the dominant search-matching paradigm.

\section{References}

Alexander, C., "The Kalai-Smorodinsky Bargaining Solution in Wage Negotiations," Journal of the Operational Research Society, 1992, 1, 779-786.

Aruoba, S.B., G. Rocheteau, and C. Waller, "Bargaining and the Value of Money," Journal of Monetary Economics, 2007, 54, 2636-2655.

Burdett, K. and M.G. Coles, "Marriage and Class*," Quarterly Journal of Economics, 1997, $112(1), 141-168$. 
- and R. Wright, "Two-sided search with nontransferable utility," Review of Economic Dynamics, 1998, 1 (1), 220-245.

Chetty, R., "A new method of estimating risk aversion," American Economic Review, 2006, 96 (5), 1821-1834.

Chun, Y., "The equal-loss principle for bargaining problems," Economics Letters, 1988, 26 (2), 103-106.

Coles, M. and A. Masters, "Optimal unemployment insurance in a matching equilibrium," Journal of Labor Economics, 2006, 24 (1), 109-138.

Fredriksson, P. and B. Holmlund, "Optimal unemployment insurance in search equilibrium," Journal of Labor Economics, 2001, 19 (2), 370-399.

Gerber, A. and T. Upmann, "Bargaining solutions at work: Qualitative differences in policy implications," Mathematical Social Sciences, 2006, 52 (2), 162-175.

Heckathorn, D., "A paradigm for bargaining and a test of two bargaining models," Behavioral Science, 1978, 23 (2), 73-85.

Hosios, A. J., "On the efficiency of matching and related models of search and unemployment," Review of Economic Studies, 1990, 57 (2), 279-298.

Jr, J.F. Nash, "The bargaining problem," Econometrica, 1950, 18 (2), 155-162.

Kalai, E., "Proportional solutions to bargaining situations: interpersonal utility comparisons," Econometrica, 1977, 45 (7), 1623-1630.

_ and M. Smorodinsky, "Other solutions to Nash's bargaining problem," Econometrica, 1975, $43(3), 513-518$.

Laroque, G. and B. Salanie, "Salaire minimum et emploi en présence de négociations salariales," Annales d'Économie et de Statistique, 2004, 3, 1-22.

Lehmann, E. and B. van der Linden, "On the optimality of search matching equilibirum when workers are risk averse," Journal of Public Economic Theory, 2007, 9, 867-884.

Manser, M. and M. Brown, "Marriage and household decision-making: A bargaining analysis," International Economic Review, 1980, 21 (1), 31-44.

McDonald, I.M. and R.M. Solow, "Wage bargaining and employment," American Economic Review, 1981, 71 (5), 896-908.

Miyagawa, E., "Subgame-perfect implementation of bargaining solutions," Games and Economic Behavior, 2002, 41 (2), 292-308. 
Mortensen, D. and C.A. Pissarides, "New developments in models of search in the labor market," in Handbook of labor economics, 1999, Volume 3, Chapter 39, Edited by O. Ashenfelter and D. Card. Amsterdam: North Holland.

Moulin, H., "Implementing the Kalai-Smorodinsky bargaining solution," Journal of Economic Theory, 1984, 33 (1), 32-45.

Nydegger, R.V., "Independent utility scaling and the Nash bargaining model," Behavioral Science, 1977, 22 (4), 283-289.

_ and G. Owen, "Two-person bargaining: An experimental test of the Nash axioms," International Journal of Game Theory, 1974, 3 (4), 239-249.

Osborne, M. and A. Rubinstein, Bargaining and Markets, Academic Press, 1990.

Petrongolo, B. and C.A. Pissarides, "Looking into the black box: A survey of the matching function," Journal of Economic Literature, 2001, 39 (2), 390-431.

Pissarides, C.A., Equilibrium unemployment theory, the MIT press, 2000.

Rogerson, R., R. Shimer, and R. Wright, "Search-theoretic models of the labor market: a survey," Journal of Economic Literature, 2005, 43 (4), 959-988.

Shimer, R., "The cyclical behavior of equilibrium unemployment and vacancies," American Economic Review, 2005, 95 (1), 25-49.

Thomson, W., "Cooperative models of bargaining," in Handbook of game theory with economic applications, 1994, Volume 2, Chapter 35, Edited by R. J. Aumann and S. Hart. Amsterdam: North Holland. 


\section{Appendix}

\section{A Wage equations}

\section{A.1 Benchmark model}

Nash solution: From (9), the foc verifies $\frac{\frac{\partial(E(w)-U)}{\partial w}}{E(w)-U}+\frac{\frac{\partial(J(w)-V)}{\partial w}}{J(w)-V}=0$ and yields the following sharing rules: $E(w)-U=J(w)-V=\frac{1}{2} S$ where $S \equiv E(w)-U+J(w)-V$ stands for the surplus of a job-worker match. Using (4) and (6) together with the sharing rules, one gets $w-r U=y-w-r V$; eq. (10) follows.

Egalitarian solution: From (11), the Egalitarian solution translates into $E(w)-U=J(w)-V$. Using (4) and (6) together with the latter expression, one gets $w-r U=y-w-r V$; eq. (12) follows.

Kalai-Smorodinsky solution: From (16), it is clear that $\frac{E(w)-U}{J(w)-V}=\frac{\widehat{E}^{e}\left(\widehat{w}^{e}\right)-U}{\widehat{J}^{f}\left(\widehat{w}^{f}\right)-V}$. Using (4), (6), (14) and (15) the LHS rewrites as $\frac{w-r U}{y-w-r V}$ and the RHS as $\frac{y-r V-r U}{y-r V-r U}$. Making use of the last two expressions, one gets $\frac{w-r U}{y-w-r V}=1$; eq. (17) follows.

\section{A.2 Model under partially transferable utility}

Nash solution: From (9), the foc verifies $\frac{\frac{\partial(E(w)-U)}{\partial w}}{E(w)-U}+\frac{\frac{\partial(J(w)-V)}{\partial w}}{J(w)-V}=0$ and simplifies to $v^{\prime}(w)(J(w)-V)=$ $(E(w)-U)$. One gets the following two sharing rules $E(w)-U=\frac{v^{\prime}(w)}{v^{\prime}(w)+1} S$ and $J(w)-V=$ $\frac{1}{v^{\prime}(w)+1} S$ with $S \equiv E(w)-U+J(w)-V$. Using (4) and (6), one gets $J(w)-V=\frac{y-w-r V}{r+s}$ and $S=\frac{y+v(w)-w-r(U+V)}{r+s}$. Combining these two relations with the sharing rules and assuming the free entry condition is satisfied yields, $w=y-\frac{1}{v^{\prime}(w)}(v(w)-r U)$. A more suitable wage equation can be derived using (3), (5) and the first order condition of the Nash program. Combining these relations and restricting to symmetric equilibria gives $r U=v(z)+\theta \gamma v^{\prime}(w)$. Replacing this in the previous expression of $w$, eq. (21) follows.

Egalitarian solution: From (11), the Egalitarian solution translates into $E(w)-U=J(w)-V$. Multiplying both sides by $r+s$, using (4), (6) and assuming that the free-entry condition, $V=0$, is satisfied, one gets: $y-w=v(w)-r U$. Next, combining (3) and (4), the expected return from unemployment verifies $r U=\frac{(r+s) v(z)+\theta q(\theta) v(w)}{r+s+\theta q(\theta)}$. Finally, combining the last two equations, eq. (22) follows.

Kalai-Smorodinsky solution: From (16), it is clear that $\frac{E(w)-U}{J(w)-V}=\frac{\widehat{E}^{e}\left(\widehat{w}^{e}\right)-U}{\widehat{J}^{f}\left(\widehat{w}^{f}\right)-V}$. Using (4), (6), (14) and (15), the LHS satisfies: $\frac{E-U}{J}=\frac{v(w)-r U}{y-w}$, and the RHS satisfies: $\frac{\widehat{E}^{e}-U}{\widehat{J}^{f}}=\frac{v(y)-r U}{y-v^{-1}(r U)}$. Combining these relations gives $(v(w)-r U)\left(y-v^{-1}(r U)\right)=(v(y)-r U)(y-w)$. Finally, eq. (23) follows as an implicit function of $r U: y-w=\frac{v(w)-r U}{v(y)-r U}\left(y-v^{-1}(r U)\right)$. 


\section{B Equilibrium analysis in the neighborhood of transferability}

The purpose of this appendix is to study the properties of the labor market equilibrium in the neighborhood of a linear utility function. This is of particular interest for the Kalai-Smorodinsky solution which is difficult to characterize in its general form. The utility function $v(w, \sigma)$ will be now be a function of a parameter $\sigma$, which will be positively linked to the risk aversion; in particular, $\sigma=0$ will correspond to the linear utility case: $v(w, 0)=w$, and we require that risk aversion be strictly increasing in $\sigma$.

Let $\zeta$ be such that $v(\zeta, \sigma)=r U$ with $r U=\frac{(r+s) v(z, \sigma)+\theta q(\theta) v(w, \sigma)}{r+s+\theta q(\theta)}$. We can rewrite this as $v(w, \sigma)-v(\zeta, \sigma)=\frac{r+s}{\theta q(\theta)}(v(\zeta, \sigma)-v(z, \sigma))$. Then, using the Job Creation condition (18), one gets:

$$
v(w, \sigma)-v(\zeta, \sigma)=\frac{y-w}{\gamma \theta}(v(\zeta, \sigma)-v(z, \sigma))
$$

The wage equations detailed in Appendix A rewrite as:

$$
\begin{aligned}
& y-w=[v(w, \sigma)-v(\zeta, \sigma)] \frac{1}{v^{\prime}(w)} \\
& y-w=v(w, \sigma)-v(\zeta, \sigma) \\
& y-w=[v(w, \sigma)-v(\zeta, \sigma)] \frac{y-\zeta}{v(y, \sigma)-v(\zeta, \sigma)}
\end{aligned}
$$

for the Nash, Egalitarian, and Kalai-Smorodinsky solutions, respectively. For consistency and notational convenience, $v^{\prime}(w)$ corresponds to $\partial v / \partial w$. For every value of the risk aversion parameter, the equilibrium is then given by a triplet $(w, \theta, \zeta)$ which is implicitly defined by a system $H_{i}(w, \theta, \zeta, \sigma)=0$ made up of equations (18), (B-1) and a wage equation (B-2) corresponding to the solution considered. $i \in\{N, E, K S\}$ stands for $N$ ash, Egalitarian and Kalai-Smorodinsky, respectively.

When $\sigma=0$, the three solutions coincide, and we will write this solution under transferability as $\left(w_{0}, \theta_{0}, \zeta_{0}\right)$; we have $H_{i}\left(w_{0}, \theta_{0}, \zeta_{0}, 0\right)=0$ for all $i$. Using the implicit function theorem, it is possible to determine the derivative of $Y=(w, \theta, \zeta)$ as a function of $\sigma$ :

$$
\left.\frac{d Y}{d \sigma}\right|_{i}=-\left(\frac{\partial H_{i}}{\partial Y}\right)^{-1} \frac{\partial H_{i}}{\partial \sigma} .
$$

By using the explicit solution in the case of transferability $\zeta_{0}=z+\gamma \theta_{0}$ and $w_{0}=\frac{1}{2}\left(y+\zeta_{0}\right)=$ $\frac{1}{2}\left(y+z+\gamma \theta_{0}\right)$, it is possible to simplify the expressions:

$$
\begin{aligned}
& \left.\frac{d w}{d \sigma}\right|_{\mathrm{N}}=\frac{(r+s) q^{\prime}\left(\theta_{0}\right)\left(\frac{\partial v}{\partial \sigma}\left(w_{0}, 0\right)-\frac{\partial v}{\partial \sigma}(z, 0)-\left(w_{0}-z\right) \frac{\partial^{2} v}{\partial \sigma \partial w}\left(w_{0}, 0\right)\right)}{q\left(\theta_{0}\right)^{2}-2(r+s) q^{\prime}\left(\theta_{0}\right)} \\
& \left.\frac{d w}{d \sigma}\right|_{\mathrm{E}}=\frac{(r+s) q^{\prime}\left(\theta_{0}\right)\left(\frac{\partial v}{\partial \sigma}\left(w_{0}, 0\right)-\frac{\partial v}{\partial \sigma}(z, 0)\right)}{q\left(\theta_{0}\right)^{2}-2(r+s) q^{\prime}\left(\theta_{0}\right)} \\
& \left.\frac{d w}{d \sigma}\right|_{\mathrm{KS}}=\frac{(r+s) q^{\prime}\left(\theta_{0}\right)\left(\frac{\partial v}{\partial \sigma}\left(w_{0}, 0\right)-\frac{\partial v}{\partial \sigma}(z, 0)-\frac{w_{0}-z}{y-\zeta_{0}}\left(\frac{\partial v}{\partial \sigma}(y, 0)-\frac{\partial v}{\partial \sigma}\left(\zeta_{0}, 0\right)\right)\right)}{q\left(\theta_{0}\right)^{2}-2(r+s) q^{\prime}\left(\theta_{0}\right)}
\end{aligned}
$$


The denominator of these three expressions is positive, as long as $q^{\prime}(\theta)<0$. With the assumption that $\frac{d v}{d \sigma}(w, \sigma)$ is increasing and concave in $w$ (this assumption is typically satisfied for both the CRRA and CARA utility functions), we have the general results that $\left.\frac{d w}{d \sigma}\right|_{\mathrm{N}}<0,\left.\frac{d w}{d \sigma}\right|_{\mathrm{E}}<0$, and $\left.\frac{d w}{d \sigma}\right|_{\mathrm{KS}}<0$

We can thus characterize the equilibrium wage in the neighborhood of linearity with the following Taylor approximation:

$$
w_{i}(\sigma)=w_{0}+\left.\sigma \frac{d w\left(w_{0}, 0\right)}{d \sigma}\right|_{i}+O\left(\sigma^{2}\right)
$$

Consequently, under partial transferability, the equilibrium wage is lower in the Nash, Egalitarian and Kalai-Smorodinsky cases than in the linear case. We can also compare the relative behavior of Nash and Kalai-Smorodinsky solutions as well as the relative behavior of Nash and Egalitarian solutions. Using (B-3), we find

$$
w_{K S}-w_{N} \equiv \frac{(r+s) q^{\prime}\left(\theta_{0}\right)\left(w_{0}-z\right)\left(\frac{\partial^{2} v}{\partial \sigma \partial w}\left(w_{0}, 0\right)-\frac{\frac{\partial v}{\partial \sigma}(y, 0)-\frac{\partial v}{\partial \sigma}\left(\zeta_{0}, 0\right)}{y-\zeta_{0}}\right)}{q\left(\theta_{0}\right)^{2}-2(r+s) q^{\prime}\left(\theta_{0}\right)}
$$

However, even with our assumptions on the utility function, it is not possible to establish the sign of this expression, and by using specific examples it can be shown that the difference can go in either directions. Thus there is no general result on the relative behaviour of the Nash and Kalai-Smorodinsky solutions that can be established without further hypotheses. Similarly, using (B-3), we find

$$
w_{E}-w_{N} \equiv \frac{(r+s) q^{\prime}\left(\theta_{0}\right)\left(w_{0}-z\right) \frac{\partial^{2} v}{\partial \sigma \partial w}\left(w_{0}, 0\right)}{q\left(\theta_{0}\right)^{2}-2(r+s) q^{\prime}\left(\theta_{0}\right)}
$$

This expression is of the sign of $q^{\prime}(\theta)$ and is, in consequence, decreasing. It follows that the wage gap between the Egalitarian and the Nash solutions is increasing in absolute terms with $\sigma$.

\section{Characterization of the Job Creation and Wage Curves}

Job Creation curve: Let $\eta \equiv \eta(\theta)=-\frac{\theta q^{\prime}(\theta)}{q(\theta)} \in[0,1]$ be the elasticity of the matching function with respect to search inputs. Differentiation of the job creation equation (18) with respect to the endogenous variables, $\theta$ and $w$, yields:

$$
d \theta=-\frac{\theta q(\theta)}{(r+s) \gamma \eta(\theta)} d w
$$

It follows that the Job Creation curve is decreasing in the $(\theta, w)$-space.

Remark 2. Assuming a Cobb-Douglas matching function as is traditional in the search-matching literature, the Job creation curve, $J c$, is decreasing and convex in the $(\theta, w)$-space.

Proof. from $\left.\frac{d \theta}{d w}\right|_{J c}=-\frac{\theta q(\theta)}{(r+s) \gamma \eta}<0$, one obtains: $\left.\frac{d^{2} \theta}{d w^{2}}\right|_{J c}=-(1-\eta) q(\theta) \frac{d \theta}{d w}>0$. 
Wage curves: First, one must note that contrary to the Job Creation curve, the shape of the wage curves are not invariant to workers' preferences. In consequence, the cases with transferable and partially transferable utility need to be distinguished.

In the former case (transferable utility), the three bargaining solutions collapse to a single wage equation. Differentiation of (10), (12) or (17) with respect to the endogenous variables, $\theta$ and $w$, together with the free-entry condition yields:

$$
d w^{j}=\frac{1}{2} \gamma d \theta \quad \text { for } \quad j \in\{N, E, K S\}
$$

It follows the wage curves are upward-sloping and linear in the $(\theta, w)$-space.

In the latter case (partially transferable utility), the shape of the wage curves are not necessarily the same. We proceed in the same manner for the three solutions below.

Remark 3. When utility is partially transferable, the Nash wage curve, $w c^{N}$, is increasing and convex in the $(\theta, w)$-space.

Proof. from $\left.\frac{d \theta}{d w}\right|_{w c^{N}}=\frac{1}{\gamma}\left(2-\frac{v^{\prime \prime}(w)[v(w)-v(z)]}{\left[v^{\prime}(w)\right]^{2}}\right)>0$, one obtains:

$\left.\frac{d^{2} \theta}{d w^{2}}\right|_{w c^{N}}=-\frac{1}{\gamma}\left[\frac{(v(w)-v(z))\left(v^{\prime \prime \prime}(w) v^{\prime}(w)^{2}-2 v^{\prime}(w) v^{\prime \prime}(w)^{2}\right)+v^{\prime \prime}(w) v^{\prime}(w)^{3}}{v^{\prime}(w)^{4}}\right]>0$.

Remark 4. When utility is partially transferable, the Egalitarian wage curve, wc ${ }^{E}$, is increasing and concave in the $(\theta, w)$-space.

Proof. from $\left.\frac{d \theta}{d w}\right|_{w c^{E}}=\frac{1}{\gamma}\left(1+v^{\prime}(w)\right)>0$, one obtains:

$\left.\frac{d^{2} \theta}{d w^{2}}\right|_{w c^{E}}=v^{\prime \prime}(w) / \gamma<0$.

Remark 5. When utility is partially transferable, the Kalai-Smorodinsky wage curve, $w c^{K S}$, is increasing in the $(\theta, w)$-space. The shape of the wage curve is however undetermined as the sign of $\left.\frac{d^{2} \theta}{d w^{2}}\right|_{w c^{K S}}$ is ambiguous ${ }^{18}$.

Proof. The equations (23) and (25) implicitly determine $w$ and $r U$ as a function of $\theta$. The system of equations is simpler if we write $\zeta$ such that $v(\zeta)=r U$, and $\lambda(\theta)=(r+s) /(r+s+\theta q(\theta))$. By using the Implicit Function theorem, the partial derivative can be calculated and proven to be positive; hence, $w c^{K S}$ is increasing in $\theta$ :

$\left.\frac{d \theta}{d w}\right|_{w c^{K S}}=-\frac{(v(w)-v(z))\left(v(w)-v(\zeta)+(w-\zeta) v^{\prime}(\zeta)\right) \lambda^{\prime}(\theta)}{(v(w)-v(\zeta))(-1+\lambda(\theta)) v^{\prime}(w)+\left(v(y)-v(\zeta)+(-w+y+(w-\zeta) \lambda(\theta)) v^{\prime}(w)\right) v^{\prime}(\zeta)}$

\section{Existence and Uniqueness:}

When utility is perfectly transferable, the wage curves have all the same shape and are all upward sloping whatever, ie. $\left.\frac{d \theta}{d w}\right|_{w^{j}}=\frac{1}{2} \gamma>0$, and the job creation is curve is downward sloping, ie. $\left.\frac{d \theta}{d w}\right|_{J c}=-\frac{\theta q(\theta)}{(r+s) \gamma \eta}<0$. Existence and uniqueness of the equilibrium follow.

\footnotetext{
${ }^{18}$ Note however that under reasonable parameter values, the wage curve is convex in the $(\theta, w)$-space.
} 
When utility is partially transferable, the wages curves differ but are all up upward sloping, ie. $\left.\frac{d \theta}{d w}\right|_{w c^{N}}=\frac{1}{\gamma}\left(2-\frac{v^{\prime \prime}(w)[v(w)-v(z)]}{\left[v^{\prime}(w)\right]^{2}}\right)>0,\left.\frac{d \theta}{d w}\right|_{w c^{E}}=\frac{1}{\gamma}\left(1+v^{\prime}(w)\right)>0,\left.\frac{d \theta}{d w}\right|_{w c^{K S}}>0$, and the job creation is curve is downward sloping, ie. $\left.\frac{d \theta}{d w}\right|_{J c}=-\frac{\theta q(\theta)}{(r+s) \gamma \eta}<0$. Existence and uniqueness of the equilibrium follow from the slopes and the values of the two curves at $z$ and at $y$.

\section{Efficiency}

\section{D.1 Benchmark model}

We restrict ourselves to the case where $r \rightarrow 0$. When utility is perfectly transferable, the social welfare function corresponds to aggregate output. This function satisfies:

$$
\Gamma=(1-u) y-\theta u \gamma
$$

The program of the benevolent planner consists in maximizing $\Gamma$ with respect to $\theta$ subject to eq. (2). The social planner chooses $\theta$ such that:

$$
(1-\eta) y-\frac{s+\eta \theta q(\theta)}{q(\theta)} \gamma=0
$$

By virtue of Proposition 1, whatever the bargaining solution, the wage rule satisfies eq. (19) when utility is transferable. Making use of this relation together with the job creation condition (18), we rewrite the decentralized equilibrium as:

$$
\frac{1}{2}(y-z)-\frac{s+\frac{1}{2} \theta q(\theta)}{q(\theta)} \gamma=0
$$

where $\eta \equiv \eta(\theta) \in[0,1]$ is the elasticity of the matching function with respect to the search inputs. Comparing the social optimum (D-10) with the decentralized equilibrium (D-11), it is simple to establish that the two relationships are equivalent if and only if $\eta=\frac{1}{2}$ and $z=0$. In other terms, job creation is socially efficient when the elasticity of the matching function is equal to the bargaining power of the workers and the unemployment benefits are nil.

\section{D.2 Model under partially transferable utility}

We restrict ourselves to the case where $r \rightarrow 0$. When utility is non transferable. The social welfare function writes:

$$
\Gamma=(1-u) v(w)+u v(z)
$$

The program of the benevolent planner consists in maximizing $\Gamma$ with respect to $\theta, w$ and $z$ subject to eq. (2) and to an aggregate resource constraint, $(1-u) w+u z=(1-u) y-\gamma \theta u$. This last constraint determines the set of feasible allocations in the economy. The Lagrangian of the problem writes:

$L=\frac{\theta q(\theta)}{s+\theta q(\theta)} v(w)+\frac{s}{s+\theta q(\theta)} v(z)+\xi\left[\frac{\theta q(\theta)}{s+\theta q(\theta)} w+\frac{s}{s+\theta q(\theta)} z-\frac{\theta q(\theta)}{s+\theta q(\theta)} y+\frac{s \gamma \theta}{s+\theta q(\theta)}\right]$ 
where $\xi$ is the multiplier associated with the second constraint. The first order conditions with respect to $\theta, w$ and $z$ are respectively given by:

$$
\begin{aligned}
\xi[q(\theta)(1-\eta) w-q(\theta)(1-\eta) z-q(\theta)(1-\eta) y+\gamma s+\gamma \theta q(\theta) \eta] & =0 \\
v^{\prime}(w)-\xi & =0 \\
v^{\prime}(z)-\xi & =0
\end{aligned}
$$

From the last two focs, it's simple to get $v^{\prime}(w)=v^{\prime}(z)$. Hence, the planner perfectly insures the workers against unemployment hazard. The first-best allocation is characterized by a perfect insurance property which implies that the workers are wage takers, ie. $w=z$. It follows that any other wage-setting mechanisms are socially inefficient. Generally-speaking, this includes the three bargaining solutions considered in the paper.

\section{E Comparative Statics}

As already mentioned, the Job Creation curve does not depend on workers' preferences. Total differentiation of (18) with respect to the endogenous variables and the key exogenous (policy) parameters yields:

$$
d \theta=\frac{\theta q(\theta)}{(r+s) \gamma \eta(\theta)}[d y-d w]-\frac{\theta}{\gamma \eta(\theta)} d \gamma-\frac{\theta}{(r+s) \eta(\theta)}[d r+d s]
$$

where $\eta(\theta) \in[0,1]$ is the elasticity of the matching function with respect to the search inputs. As regards the wage curves, we once again contrast two cases, ie. transferable and non transferable utility. The wage equations rewrite for $j \in\{N, E, K S\}$ under the following generic form:

$$
A^{j}=y-w+\theta \gamma
$$

where $j$ stands for the Nash $(N)$, the Egalitarian $(E)$ and the Kalai-Smorodinsky $(K S)$, solutions respectively. Differentiation of (E-14) wage curve with respect to to the endogenous variables and the key exogenous (policy) parameters yields:

$$
\left[1+\frac{\partial A^{j}}{\partial w}\right] d w=\left[\gamma-\frac{\partial A^{j}}{\partial \theta}\right] d \theta+\left[1-\frac{\partial A^{j}}{\partial y}\right] d y-\frac{\partial A^{j}}{\partial z} d z+\left[\theta-\frac{\partial A^{j}}{\partial \gamma}\right] d \gamma
$$

\section{E.1 Benchmark model}

When utility is perfectly transferable, the three bargaining solutions collapse to a single wage equation. We have $A^{j}=w-z$ for $j \in\{N, E, K S\}$ and eq. (E-14) rewrites as $w-z=y-w+\theta \gamma$. For the three solutions, we get $\frac{\partial A^{j}}{\partial w}=1, \frac{\partial A^{j}}{\partial z}=-1$ and $\frac{\partial A^{j}}{\partial \theta}=\frac{\partial A^{j}}{\partial y}=\frac{\partial A^{j}}{\partial \gamma}=0$. Using the partial derivatives together with (E-13) and (E-15), the comparative-statics results follow. The table below summarizes the results. 


\begin{tabular}{clccccc}
\hline \hline Endogenous variable & Bargaining solution & $y$ & $z$ & $\gamma$ & $r$ & $s$ \\
\hline \multirow{3}{*}{$\theta$ (tightness) } & Nash & + & - & - & - & - \\
& Egalitarian & + & - & - & - & - \\
& Kalai-Smorodinsky & + & - & - & - & - \\
\hline \multirow{3}{*}{$w$ (wage) } & Nash & + & + & - & - & - \\
& Egalitarian & + & + & - & - \\
& Kalai-Smorodinsky & + & + & - & - \\
\hline \hline
\end{tabular}

Table 4: Comparative statics results when utility is perfectly transferable.

\section{E.2 Model under partially transferrable utility}

When utility is partially transferable, the three bargaining solutions are no longer equivalent. We proceed with the analysis of the Nash, the Egalitarian and the Kalai-Smorodinsky solutions separately. In the first two cases, the comparative statics results established when utility is perfectly transferable readily extend to the case of partially transferable utility. Things turn out be to be a little more complicated as regards the KS solution which depends on $r U$ (see below).

Nash solution: Under the Nash solution, we have $A^{N}=(v(w)-v(z)) / v^{\prime}(w)$ and eq. (E-14) rewrites as $(v(w)-v(z)) / v^{\prime}(w)=y-w+\gamma \theta$. We get $\frac{\partial A^{N}}{\partial w}=\left[1-\frac{v^{\prime \prime}(w)}{v^{\prime}(w)} \frac{v(w)-v(z)}{v^{\prime}(w)}\right]>0, \frac{\partial A^{N}}{\partial z}=$ $-\frac{v^{\prime}(z)}{v^{\prime}(w)}<0$ and $\frac{\partial A^{N}}{\partial \theta}=\frac{\partial A^{N}}{\partial y}=\frac{\partial A^{N}}{\partial \gamma}=0$. Using the partial derivatives together with (E-13) and (E-15), the comparative-statics results follow. Table 5 summarizes the results.

Egalitarian solution: Under the Egalitarian solution we have $A^{E}=(v(w)-v(z))$ and eq. $\left(\right.$ E-14) rewrites as $(v(w)-v(z))=y-w+\gamma \theta$. We get $\frac{\partial A^{E}}{\partial w}=v^{\prime}(w)>0, \frac{\partial A^{E}}{\partial z}=-v^{\prime}(z)<0$ and $\frac{\partial A^{E}}{\partial \theta}=\frac{\partial A^{E}}{\partial y}=\frac{\partial A^{E}}{\partial \gamma}=0$. Using the partial derivatives together with (E-13) and (E-15), the comparative-statics results follow. Table 5 summarizes the results.

Kalai-Smorodinsky solution: Under the KS solution we have $A^{K S}=\frac{(v(w)-r U)\left(y-v^{-1}(r U)\right)+\theta \gamma(v(y)-r U)}{v(y)-r U}$ and eq. (E-14) rewrites as $\frac{(v(w)-r U)\left(y-v^{-1}(r U)\right)+\theta \gamma(v(y)-r U)}{v(y)-r U}=y-w+\gamma \theta$. In its general form, the $\mathrm{KS}$ solution is not tractable. In order to derive analytical results, we restrict our analysis to the neighborhood of the linearity which make the results appropriate for a low degree of risk aversion. We consider now the following approximation of the utility function: $v(w)=w-\sigma w^{2}+o(\sigma)$. Solving for $\zeta$ in (B-1), then for $w$ in (B-2), and taking the Taylor series expansion for $\sigma$ small, we get:

$$
H(w, \theta, y, \gamma, z, r, s, \sigma)=\left\{\begin{array}{c}
w-\left(\frac{1}{2}(y+z+\gamma \theta)-\frac{\sigma}{8}(y-z+\gamma \theta)^{2}\right) \\
\frac{r+s}{q(\theta)}-\frac{y-w}{\gamma}
\end{array}\right\}
$$

Using the implicit function theorem and matrix notations with $x=(y, \gamma, z, r, s)$ et $X=(w, \theta)$, we have:

$$
\frac{d X}{d x}=-\left(\frac{\partial H}{\partial X}\right)^{-1} \frac{\partial H}{\partial x}
$$


Let $\Delta=-\left[(-2+\sigma(y+\gamma \theta-z)) q(\theta)^{2}+4 r q^{\prime}(\theta)\right]$, then we get:

$$
\begin{aligned}
\frac{d w}{d y} & =\frac{1}{\Delta}(\gamma \theta+\eta(y-w))(2-\sigma(y+\gamma \theta-z)) \\
\frac{d w}{d z} & =\frac{1}{\Delta} \eta(y-w)(2-\sigma(y+\gamma \theta-z)) \\
\frac{d w}{d \gamma} & =\frac{1}{\Delta}(\eta-1)(y-w)(2-\sigma(y+\gamma \theta-z)) \\
\frac{d w}{d r}=\frac{d w}{d s} & =-\frac{1}{r \Delta} \gamma \theta(y-w)(2-\sigma(y+\gamma \theta-z)) \\
\frac{d \theta}{d y} & =\frac{1}{\Delta} \theta(2+\sigma(y+\gamma \theta-z)) \\
\frac{d \theta}{d z} & =-\frac{1}{\gamma \Delta} \theta(1-\eta)(y-w)(2-\sigma(y+\gamma \theta-z)) \\
\frac{d \theta}{d \gamma} & =-\frac{1}{\Delta} \theta(2 \gamma \theta+4(y-w)+\sigma \gamma \theta(y+\gamma \theta-z)) \\
\frac{d \theta}{d r}=\frac{d \theta}{d s} & =-\frac{1}{r \Delta} \theta(y-w)
\end{aligned}
$$

Hence if $\frac{\sigma}{2}(y+\gamma \theta-z)<1$, we know that $\Delta>0$ since $q^{\prime}(\theta)<0$ and we have $\frac{d w}{d y}>0, \frac{d w}{d z}>0$, $\frac{d w}{d \gamma}<0, \frac{d w}{d r}<0$ and $\frac{d w}{d s}<0$, as well as $\frac{d \theta}{d y}>0, \frac{d \theta}{d z}<0, \frac{d \theta}{d \gamma}<0, \frac{d \theta}{d r}<0$ and $\frac{d \theta}{d s}<0$. As the derivation requires $\sigma \simeq 0$, the condition will hold for $\sigma$ that is small enough.

\begin{tabular}{clccccc}
\hline \hline Endogenous variable & Bargaining solution & $y$ & $z$ & $\gamma$ & $r$ & $s$ \\
\hline \multirow{3}{*}{$\theta$ (tightness) } & Nash & + & - & - & - & - \\
& Egalitarian & + & - & - & - & - \\
& Kalai-Smorodinsky & + & - & - & - & - \\
\hline \multirow{3}{*}{$w$ (wage) } & Nash & + & + & - & - & - \\
& Egalitarian & + & + & - & - \\
& Kalai-Smorodinsky & + & + & - & - \\
\hline \hline
\end{tabular}

Table 5: Comparative statics results when utility is partially transferable utility; in the neighborhood of linearity for the Kalai-Smorodinsky solution. 


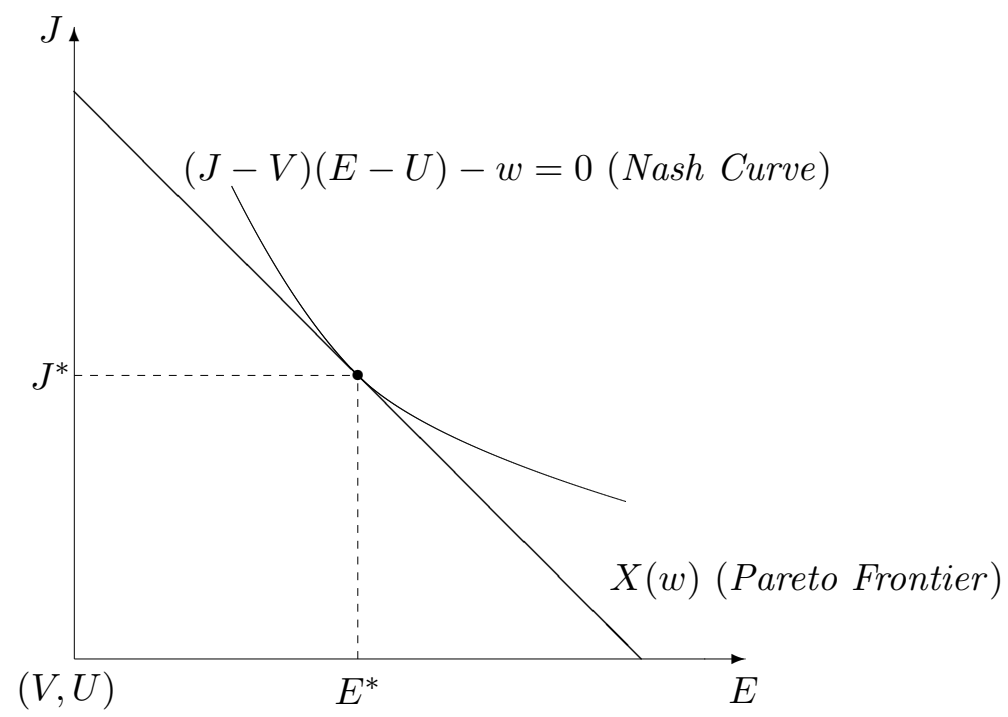

Figure 1: Nash solution with transferable utility.

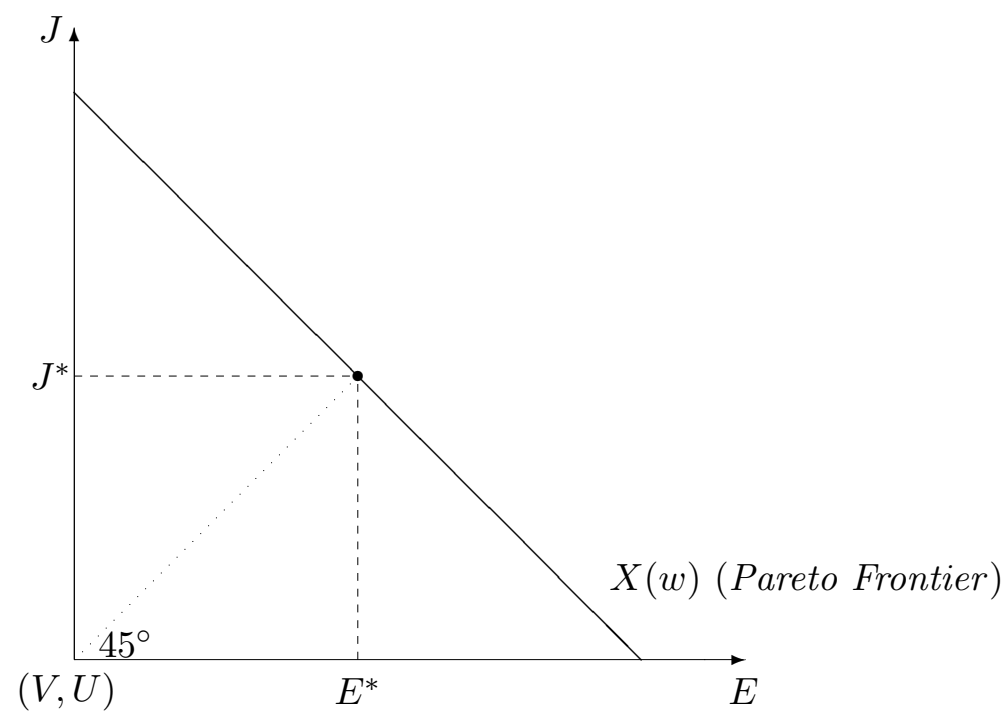

Figure 2: Egalitarian solution with transferable utility. 


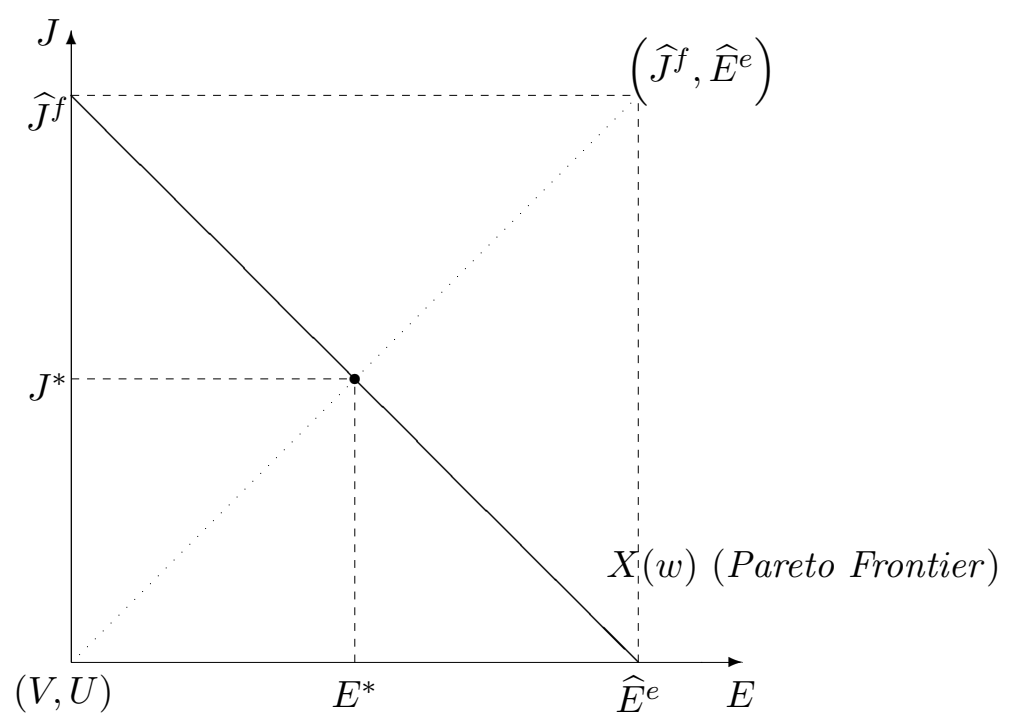

Figure 3: Kalai-Smorodinsky solution with transferable utility.

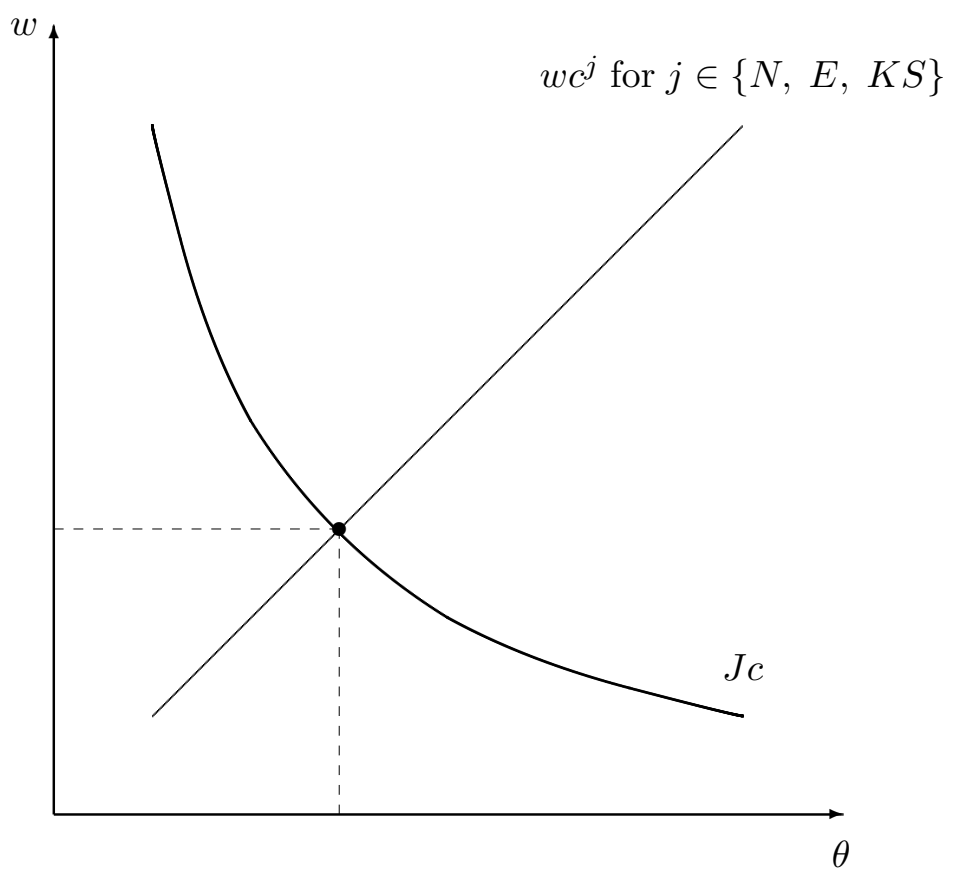

Figure 4: Labor market equilibria with transferable utility; $\mathrm{N}$ for Nash, E for Egalitarian, KS for Kalai-Smorodinsky. 


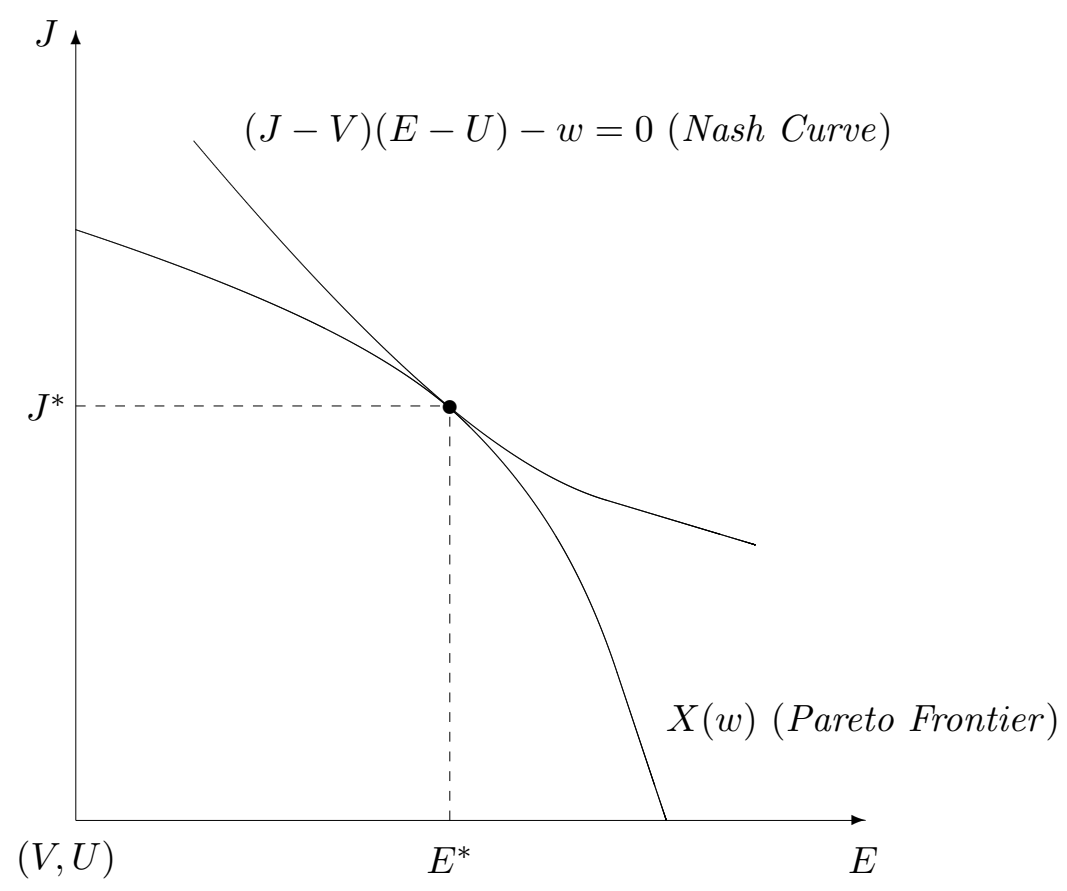

Figure 5: Nash solution with partially transferable utility.

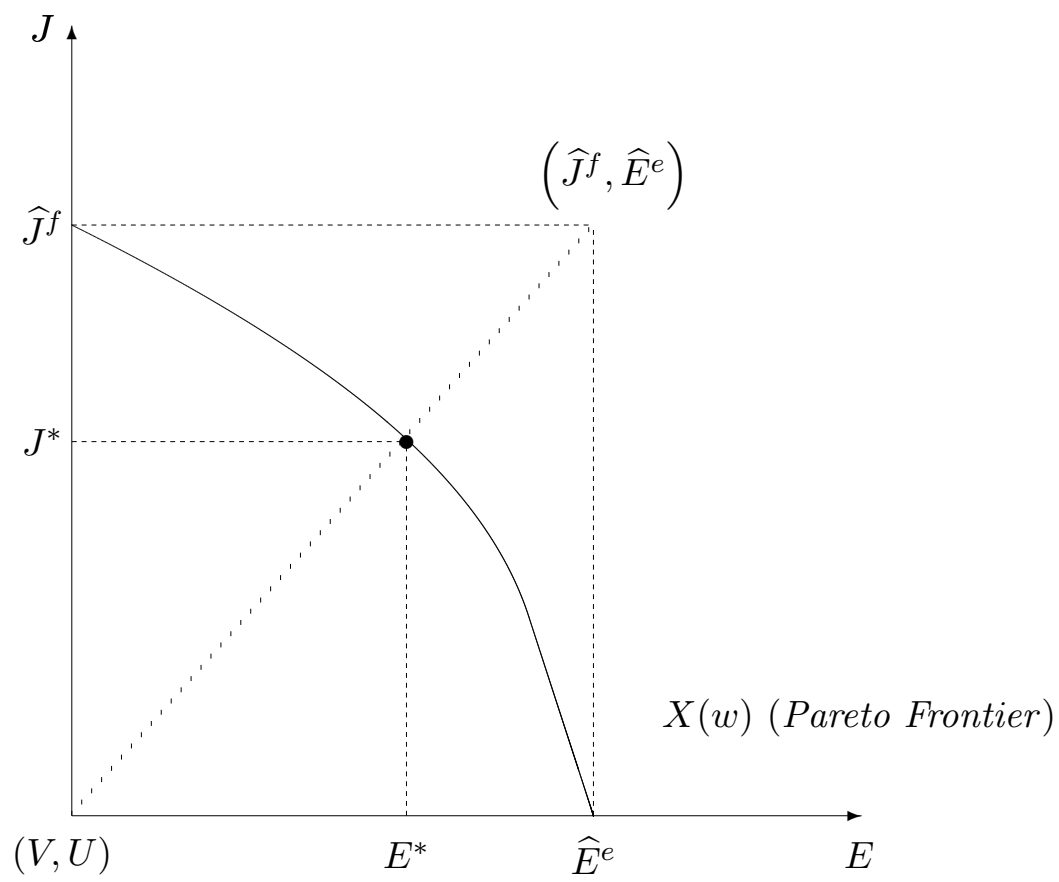

Figure 6: Kalai-Smorodinsky solution with partially transferable utility. 


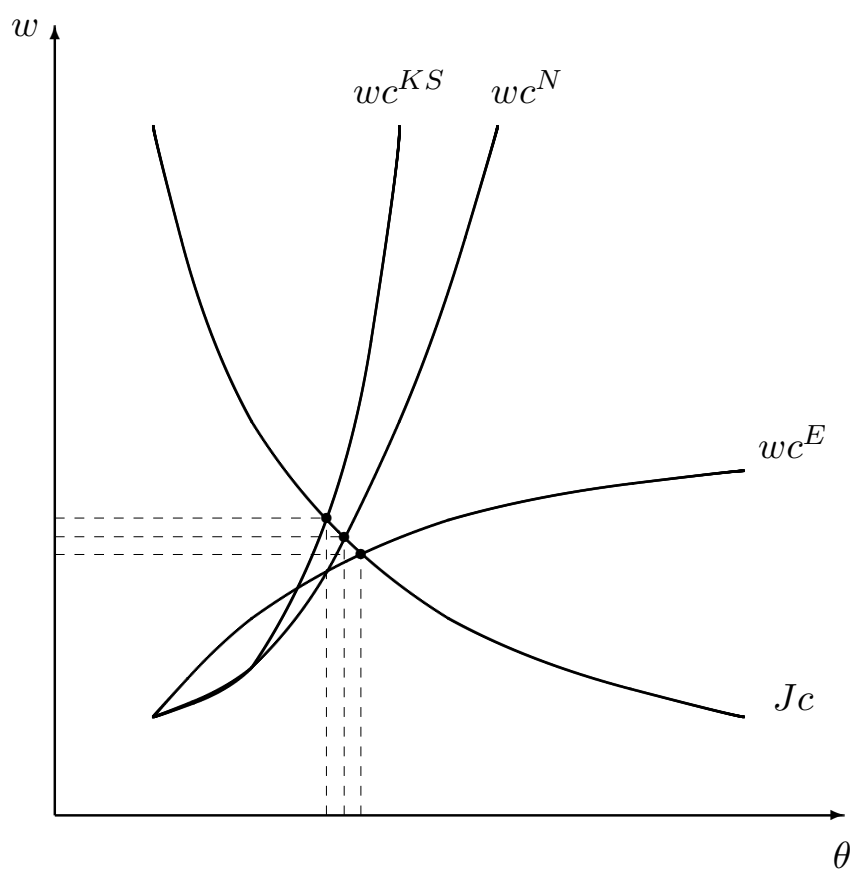

Figure 7: Labor market equilibria with partially transferable utility; $\mathrm{N}$ for Nash, E for Egalitarian, KS for Kalai-Smorodinsky; Wages and Tightness are ranked as shown in Table 2; Hierarchy in the endogenous variables for the three solutions may be mathematically established in the neighborhood of linearity. Proofs are available from the authors upon request.
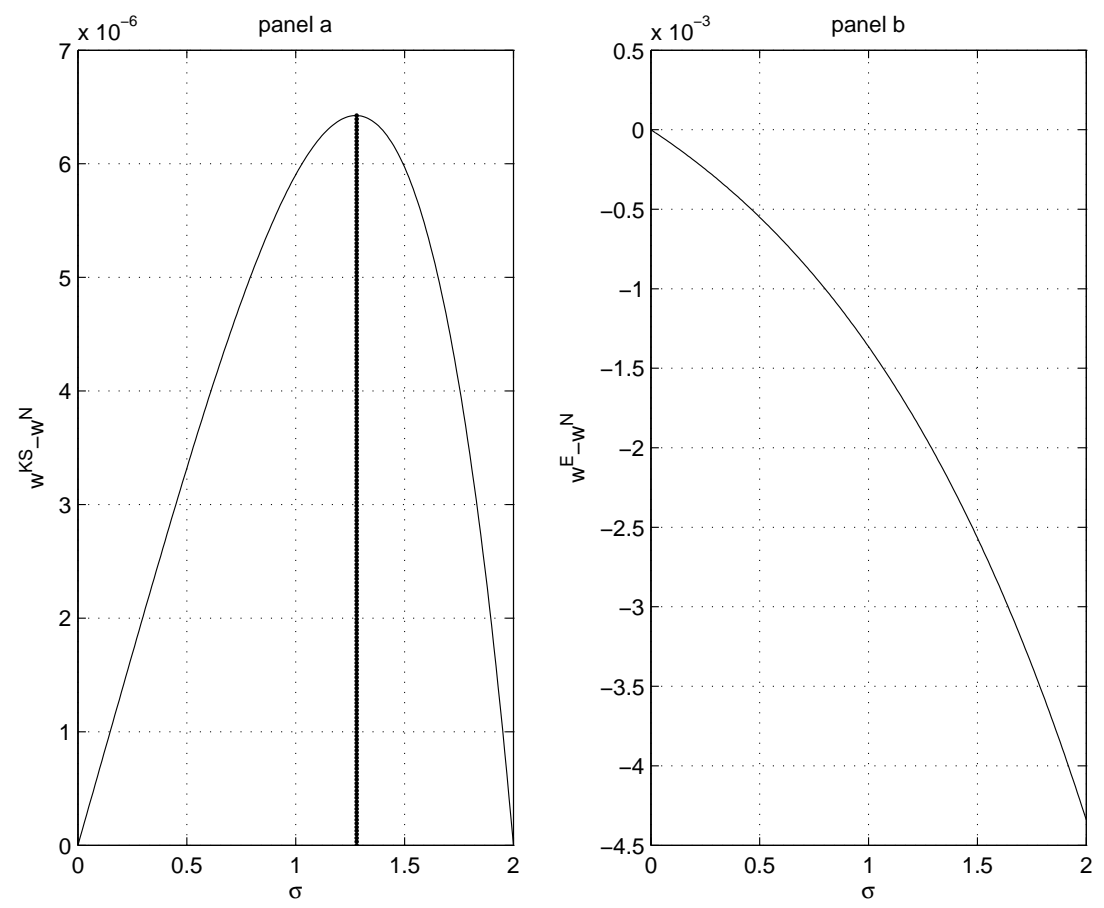

Figure 8: Difference between the Nash and the Kalai-Smorodinsky solutions (panel a) and the Nash and the Egalitarian solutions (panel b) under CRRA specification over the range $\sigma \in[0,2]$. On the first panel, the difference is maximized for $\sigma=1.28$. 